\title{
Relation between Energetic and standard Geodesic Acoustic Modes
}

\author{
Jean-Baptiste Girardo, ${ }^{1}$ David Zarzoso, ${ }^{2}$ Rémi Dumont, ${ }^{1}$ Xavier Garbet, ${ }^{1}$ Yanick Sarazin, ${ }^{1}$ and Sergei Sharapov ${ }^{3}$ \\ ${ }^{1)}$ CEA, IRFM, F-13108 Saint-Paul-lez-Durance, France. \\ ${ }^{2)}$ Max-Planck Institut für Plasmaphysik, 85748 Garching, Germany. \\ ${ }^{3)}$ CCFE, Culham Science Centre, Abingdon, OX14 3DB, UK.
}

(Dated: July 24, 2014)

Geodesic Acoustic Modes (GAMs) are electrostatic, axisymmetric modes which are non-linearly excited by turbulence. They can also be excited linearly by fast-particles; they are then called Energetic-particle-driven GAMs (EGAMs). Do GAMs and EGAMs belong to the same mode branch? Through a linear, analytical model, in which the fast particles are represented by a Maxwellian bump-on-tail distribution function, we find that the answer depends on several parameters. For low values of the safety factor $q$ and for high values of the fast ion energy, the EGAM originates from the GAM. On the contrary, for high values of $q$ and for low values of the fast ion energy, the GAM is not the mode which becomes unstable when fast particles are added: the EGAM then originates from a distinct mode, which is strongly damped in the absence of fast particles. The impact of other parameters is further explored: ratio of the ion temperature to the electron temperature, width of the fast particle distribution, mass and charge of the fast ions. The ratio between the EGAM and the GAM frequencies was found in experiments (DIII-D) and in non-linear numerical simulations (code GYSELA) to be close to 1/2: the present analytical study allows one to recover this ratio.

\section{INTRODUCTION}

Turbulent transport in tokamaks is a major hurdle on the road to burning plasma, as it involves high losses of energy from the core of the reactors. Geodesic Acoustic Modes ${ }^{1}$ (GAMs) are known to have interactions with turbulence $^{2-4}$. In thermal plasma, those modes are damped. It was found, in a JET experiment with Ion Cyclotron Resonant Heating $(\mathrm{ICRH})$, that $\mathrm{n}=0$ modes identified $^{5,6}$ as Global GAMs could be driven unstable by trapped energetic ions. This observation led to the idea of controlling the GAMs, and perhaps plasma turbulence coupled to the GAMs, with energetic particles. Later, an experiment on DIII-D with counter-current Neutral Beam Injection (NBI) revealed modes, somewhat similar to those observed on JET, but at a frequency twice lower than the characteristic GAM frequency ${ }^{7,8}$. Studies $^{9-11}$ aiming at explaining this experiment have found new modes called Energetic-particle-driven GAMs (EGAMs), and investigated them. Those EGAMs have also been detected $^{12}$ in the $5 \mathrm{D}$ gyrokinetic code GYSELA ${ }^{13-15}$, at about half the expected GAM frequency, like in the DIIID experiment ${ }^{7}$. Due to their similarity with GAMs, the EGAMs are good candidates for reducing turbulent transport, thanks to vortex shearing. However, recent numerical simulations suggest that the impact of EGAMs on turbulence may not always be as positive as expected, and requires further investigation ${ }^{16}$. EGAMs can also be responsible for fast ion losses under certain circumstances ${ }^{17}$.

The relation between GAMs and EGAMs is somewhat unclear and remains to be explained: it is the purpose of this paper. Through a linear, analytical model, the relation between GAMs and EGAMs is investigated. The fast particles are modelled by a Maxwellian bump-on-tail distribution function, in which only deeply passing ions are retained.
The link between GAMs and EGAMs is found to depend on several parameters: the safety factor $q$, the fast ion parallel velocity $\bar{u}_{\|}$normalised to the thermal velocity, the ratio $\tau_{k}$ of the fast ion distribution width to the bulk ion temperature, the ratio $T_{t} / T_{e}$ of the bulk ion temperature to the electron temperature, the mass $m_{k}$ and the charge number $Z_{k}$ of the fast ions. Those parameters are explored in the following ranges: $1 \leq q \leq 3$; $2 \leq \bar{u}_{\|} \leq 4 ; 0.1 \leq \tau_{k} \leq 2$ and $0.5 \leq T_{t} / T_{e} \leq 2$; while the masses and charge numbers correspond to those of Hydrogen, Deuterium, Tritium and Helium 3. For low values of $q$ and $m_{k}$; for high values of $\bar{u}_{\|}, \tau_{k}$ and $T_{t} / T_{e}$, the EGAM originates from the GAM. On the contrary, for high values of $q$ and $m_{k}$; for low values of $\bar{u}_{\|}, \tau_{k}$ and $T_{t} / T_{e}$, the GAM is not the mode which becomes unstable when fast particles are added: the EGAM then originates from a distinct mode, which is strongly damped in the absence of fast particles. Note that such a split in the nature of the EGAM depending on the parameters of the plasma was already exposed in Ref. 9. The present analysis gives further information about this split; the origin of the two branches is clarified. In particular, it is here established that one of the two branches (the so called Landau $E G A M)$ originates from a branch of Landau modes which already exist and are stable in the absence of fast particles. When kinetic particles are added, one of the stable Landau modes sees its frequency rise progressively to the upper part of the complex plane, and turns into an excited mode. In addition, the present model takes into account the possibility of having different species for thermal ions on the one hand, and kinetic particles on the other hand. This leads to interesting results, in particular regarding the excitation threshold of the EGAM, which is found to be very low under certain circumstances (figure 10).

The analysis carried out in this paper allows one to better understand the ways in which the real frequency and the growth rate of the GAMs and EGAMs can be modi- 
fied. This is important in particular to better apprehend the interaction of GAMs and EGAMs with turbulence.

After exposing the linear, analytical model which has been used in the present work to study the link between GAMs and EGAMs, we detail the results which were obtained through numerical resolution of the dispersion relation. Those results are then discussed.

\section{MODEL}

\section{A. Equations}

An electrostatic description of the plasma is used: the magnetic field $\mathbf{B}=I(\psi) \boldsymbol{\nabla} \varphi+\boldsymbol{\nabla} \varphi \times \boldsymbol{\nabla} \psi$ is independent of time $t . \varphi$ is the toroidal angle, $\psi$ is the magnetic poloidal flux normalised to $2 \pi$, and the poloidal angle is written $\theta$. $(\psi, \theta, \varphi)$ constitutes a direct set of coordinates in which the safety factor $q$ depends on $\psi$ only. The aspect ratio $\frac{R_{0}}{a}$, where $a$ is the minor radius of the tokamak and $R_{0}$ the major radius on the magnetic axis, is assumed to be large. The distribution functions of the thermal ion and kinetic ion gyro-centres are called, respectively, $f_{t}$ and $f_{k}$ ( $t$ for thermal and $k$ for kinetic). The total ion gyro-centre distribution function $f$ thus reads: $f=f_{t}+$ $f_{k}$. Since most equations for $f_{t}$ and $f_{k}$ are identical, the subscript $s$ (for species) will be used instead of $t$ or $k$ whenever possible, and only one equation will be written. The gyrokinetic equation for the ion distribution functions $f_{s}$ reads:

$$
\frac{\partial f_{s}}{\partial t}+\mathbf{v}_{d, s} \cdot \nabla_{\perp} f_{s}+v_{\|} \nabla_{\|} f_{s}+\frac{d \varepsilon_{s}}{d t} \frac{\partial f_{s}}{\partial \varepsilon_{s}}=0
$$

where the subscript $s$ stands for species $s$ and can be replaced with either $t$ or $k, \varepsilon_{s}=\frac{1}{2} m_{s} v_{\|}^{2}+\mu_{s} B$ is the kinetic energy, $\mu_{s}=\frac{m_{s} v_{\perp}^{2}}{2 B}$ is the magnetic moment and
$B$ is the intensity of the magnetic field. $\mathbf{v}_{d, s}$ is the drift velocity comprising the electric drift $\frac{\mathbf{b} \times \boldsymbol{\nabla} \phi}{B}$, the gradient drift $\frac{\mu_{s} B}{e_{s} B} \frac{\mathbf{b} \times \boldsymbol{\nabla} B}{B}$ and the curvature drift which reads $\frac{m_{s} v_{\|}^{2}}{e_{s} B} \frac{\mathbf{b} \times \boldsymbol{\nabla} B}{B}$ in the low $\beta$ limit. $e_{s}$ stands for the electric charge of the species $s, \mathbf{b}$ is the unitary vector directed along the magnetic field, $\beta$ is the ratio of the kinetic energy to the magnetic energy. In the present work, the long wavelength limit is considered, so that the gyroaveraged electric potential is simply replaced by the electric potential $\phi$ itself. $\mu_{s}$ is an adiabatic motion invariant: $\frac{d \mu_{s}}{d t}=0$. The kinetic energy varies according to the electric energy transfers: $\frac{d \varepsilon_{s}}{d t}=-e_{s} \mathbf{v}_{s} \cdot \nabla \phi$.

The equilibrium electric field is assumed to be small, so that $\phi$ represents the perturbed part only of the electrostatic potential. The electrons are considered adiabatic:

$$
\delta n_{e}=n_{e q} \frac{e}{T_{e}}\left(\phi-\langle\phi\rangle_{F S}\right)
$$

where $n_{e q}$ is the equilibrium density of electrons, $\delta n_{e}$ is the perturbed density of electrons, $T_{e}$ is the electron temperature, $e$ is the elementary Coulomb charge and $\langle\cdot\rangle_{F S}$ stands for the average on a magnetic flux surface.

The distribution function of the ions of species $s$ is decomposed into an equilibrium part $F_{s}$ and a perturbed part $\delta f_{s}$ :

$$
f_{s}=F_{s}\left(1+\delta f_{s}\right)
$$

With those notations, the overall ion distribution function $f$ reads:

$$
f=\sum_{s} f_{s}=f_{t}+f_{k}=F_{t}\left(1+\delta f_{t}\right)+F_{k}\left(1+\delta f_{k}\right)
$$

The quasi-neutrality equation $\delta n_{e}=Z_{t} \delta n_{t}+Z_{k} \delta n_{k}$ reads (see Appendix):

$$
n_{e q} \frac{e}{T_{e}}\left(\phi-\langle\phi\rangle_{F S}\right)=\sum_{s \in\{t, k\}}\left[\operatorname{div}\left(\frac{m_{s} n_{s}}{e B^{2}} \nabla_{\perp} \phi\right)+Z_{s} \int F_{s} \delta f_{s} d v^{3}\right]
$$

where, for species $s, Z_{s}$ is the charge number, $\delta n_{s}$ is the perturbed density, and $n_{s}$ is the equilibrium density. The $n_{s}$ verify: $n_{e q}=Z_{t} n_{t}+Z_{k} n_{k}$. The electrostatic potential is from here onwards normalized to $\frac{T_{t}}{e}$, where $T_{t}$ is the thermal ion temperature. The gyrokinetic equation is linearised in $\delta f_{s}$ and $\phi$; the equilibrium radial gradients are neglected. The computation is executed up to order 1 in $\rho_{*}=\rho_{c} / a$, where $\rho_{c}$ is the ion Larmor radius.

In the case of GAMs and EGAMs, two Fourier modes are predominantly excited: $(m, n)=(0,0)$ and $(1,0)$ where $m$ is the poloidal mode number, and $n$ is the toroidal mode number. For the density and for the elec- trostatic potential $(1,0)$ modes, the $\sin \theta$ part is predominant over the $\cos \theta$ part, where $\theta$ is counted from the equatorial plane.

\section{B. GAM Dispersion Relation}

The bulk plasma particles at equilibrium are represented by a local Maxwellian distribution:

$$
F_{t}=n_{t}\left(\frac{m_{t}}{2 \pi T_{t}}\right)^{\frac{3}{2}} e^{-\frac{\varepsilon_{t}}{T_{t}}}
$$


where the subscript $t$ stands for thermal.

In the absence of fast particles, with $Z_{t}=1$, the dispersion relation of the GAM reads:

$$
\frac{1}{q^{2}}+A_{1}\left(\Omega_{t}\right)-\frac{N_{1}\left(\Omega_{t}\right)^{2}}{D_{1}\left(\Omega_{t}\right)}=0
$$

where:

$$
\begin{aligned}
& A_{1}\left(\Omega_{t}\right)=\mathcal{Z}\left(\Omega_{t}\right)\left(\Omega_{t}^{3}+\Omega_{t}+\frac{1}{2 \Omega_{t}}\right)+\Omega_{t}^{2}+\frac{3}{2} \\
& N_{1}\left(\Omega_{t}\right)=\mathcal{Z}\left(\Omega_{t}\right)\left(\Omega_{t}^{2}+\frac{1}{2}\right)+\Omega_{t} \\
& D_{1}\left(\Omega_{t}\right)=\mathcal{Z}\left(\Omega_{t}\right) \Omega_{t}+1+\frac{T_{t}}{T_{e}}
\end{aligned}
$$

$$
\Omega_{t}=q R \sqrt{\frac{m_{t}}{2 T_{t}}} \omega
$$

and where $\omega$ is the frequency of the mode, $q$ is the safety factor, $R$ is the major radius, and $\mathcal{Z}$ is the plasma dispersion function ${ }^{18}$. This dispersion relation is the same as the one obtained in Ref. 19.

\section{EGAM Dispersion Relation}

The bulk plasma particles at equilibrium are still represented by a local centred Maxwellian distribution, while the fast particles are represented by a shifted Maxwellian distribution (called in this article bump-on-tail distribution) in parallel velocity:

$$
F_{k}=\frac{n_{k}}{2}\left(\frac{m_{k}}{2 \pi T_{t} \tau_{k}}\right)^{\frac{3}{2}}\left[e^{-\frac{m_{k}\left(v_{\|}-\bar{v}_{\|}\right)^{2}+2 \mu_{k} B}{2 T_{t} \tau_{k}}}+e^{-\frac{m_{k}\left(v_{\|}+\bar{v}_{\|}\right)^{2}+2 \mu_{k} B}{2 T_{t} \tau_{k}}}\right]
$$

where the subscript $k$ stands for kinetic and $\bar{v}_{\|}$is the position of the bump of the distribution function on the $v_{\|}$axis. It is to be noted that the distribution function of the fast particles is a centred Maxwellian in perpendicular velocity, and that it is even in parallel velocity: consequently, no parallel momentum is injected ${ }^{20}$. In addition, the distribution function (8) can be recast as a function of motion invariants only (see Appendix). $\tau_{k}$ is a temperature-like, dimensionless parameter which de- scribes the width of the bumps in $v_{\|}$.

In the presence of fast particles described by such a bump-on-tail distribution function, the dispersion relation of the EGAM reads:

$$
\frac{1}{q^{2}}+A_{1}\left(\Omega_{t}\right)+A_{2}\left(\Omega_{k}\right)-\frac{\left[N_{1}\left(\Omega_{t}\right)+N_{2}\left(\Omega_{k}\right)\right]^{2}}{D_{1}\left(\Omega_{t}\right)+D_{2}\left(\Omega_{k}\right)}=0
$$

where:

$$
\begin{aligned}
A_{1}\left(\Omega_{t}\right)= & \frac{n_{e q}}{n_{t}+n_{k} \frac{m_{k}}{m_{t}}} \frac{n_{t}}{n_{e q}}\left\{\mathcal{Z}\left(\Omega_{t}\right)\left[\Omega_{t}^{3}+\Omega_{t}+\frac{1}{2 \Omega_{t}}\right]+\Omega_{t}^{2}+\frac{3}{2}\right\} \\
A_{2}\left(\Omega_{k}\right)= & \frac{m_{k}}{m_{t}} \frac{n_{e q}}{n_{t}+n_{k} \frac{m_{k}}{m_{t}}} \frac{n_{k}}{2 n_{e q}}\left\{\left[\mathcal{Z}\left(\frac{\Omega_{k}-\bar{u}_{\|}}{\sqrt{\tau_{k}}}\right)\left[1-\frac{\bar{u}_{\|}}{\Omega_{k}}\right]+\mathcal{Z}\left(\frac{\Omega_{k}+\bar{u}_{\|}}{\sqrt{\tau_{k}}}\right)\left[1+\frac{\bar{u}_{\|}}{\Omega_{k}}\right]\right]\left[\frac{\Omega_{k}^{3}}{\tau_{k}^{3 / 2}}+\frac{\Omega_{k}}{\sqrt{\tau_{k}}}+\frac{\sqrt{\tau_{k}}}{2 \Omega_{k}}\right]\right. \\
& \left.+2 \frac{\Omega_{k}^{2}}{\tau_{k}}+3-\frac{\bar{u}_{\|} \sqrt{\tau_{k}}}{\Omega_{k}^{2}} \mathcal{Z}\left(\frac{\bar{u}_{\|}}{\sqrt{\tau_{k}}}\right)\right\} \\
N_{1}\left(\Omega_{t}\right)= & Z_{t} \sqrt{\frac{n_{e q}}{n_{t}+n_{k} \frac{m_{k}}{m_{t}}}} \frac{n_{t}}{n_{e q}}\left\{\mathcal{Z}\left(\Omega_{t}\right)\left[\Omega_{t}^{2}+\frac{1}{2}\right]+\Omega_{t}\right\} \\
N_{2}\left(\Omega_{k}\right)= & Z_{k} \frac{1}{\sqrt{\tau_{k}} \sqrt{\frac{m_{k}}{m_{t}}}} \sqrt{\frac{n_{e q}}{n_{t}+n_{k} \frac{m_{k}}{m_{t}}}} \frac{n_{k}}{2 n_{e q}}\left\{\left[\mathcal{Z}\left(\frac{\Omega_{k}-\bar{u}_{\|}}{\sqrt{\tau_{k}}}\right)\left[1-\frac{\bar{u}_{\|}}{\Omega_{k}}\right]+\mathcal{Z}\left(\frac{\Omega_{k}+\bar{u}_{\|}}{\sqrt{\tau_{k}}}\right)\left[1+\frac{\bar{u}_{\|}}{\Omega_{k}}\right]\right]\left[\frac{\Omega_{k}^{2}}{\tau_{k}}+\frac{1}{2}\right]\right. \\
& \left.+2 \frac{\Omega_{k}}{\sqrt{\tau_{k}}}-\frac{\bar{u}_{\|}}{\Omega_{k}} \mathcal{Z}\left(\frac{\bar{u}_{\|}}{\sqrt{\tau_{k}}}\right)\right\} \\
D_{1}\left(\Omega_{t}\right)= & Z_{t}^{2} \frac{n_{t}}{n_{e q}}\left\{\mathcal{Z}\left(\Omega_{t}\right) \Omega_{t}+1\right\}+\frac{T_{t}}{T_{e}}
\end{aligned}
$$




$$
D_{2}\left(\Omega_{k}\right)=\frac{Z_{k}^{2}}{\tau_{k}} \frac{n_{k}}{2 n_{e q}}\left\{\mathcal{Z}\left(\frac{\Omega_{k}-\bar{u}_{\|}}{\sqrt{\tau_{k}}}\right) \frac{\Omega_{k}}{\sqrt{\tau_{k}}}\left[1-\frac{\bar{u}_{\|}}{\Omega_{k}}\right]+\mathcal{Z}\left(\frac{\Omega_{k}+\bar{u}_{\|}}{\sqrt{\tau_{k}}}\right) \frac{\Omega_{k}}{\sqrt{\tau_{k}}}\left[1+\frac{\bar{u}_{\|}}{\Omega_{k}}\right]+2\right\}
$$

and where:

$$
\begin{aligned}
& \Omega_{k}=q R \sqrt{\frac{m_{k}}{2 T_{t}}} \omega \\
& \bar{u}_{\|}=\sqrt{\frac{m_{k}}{2 T_{t}}} \bar{v}_{\|}
\end{aligned}
$$

The details of the derivation of this dispersion relation can be found in the Appendix. The dispersion relation (7) is recovered in the absence of fast particles, i.e. for $n_{k}=0$, and for $Z_{t}=1$.

\section{RESULTS}

\section{A. Impact of the safety factor $q$ on the link between GAMs and EGAMs}

The dispersion relation (9) is numerically solved for various parameters of the bulk plasma and of the fast particles. Calling $D$ the left hand-side of that relation, we look for the zeros of $D$ as a function of $\Omega_{t}, \Omega_{k}$ being itself a linear function of $\Omega_{t}: \Omega_{k}=\sqrt{\frac{m_{k}}{m_{t}}} \Omega_{t}$. On figures 1 and 2 are shown the contour representations of $\left|D\left(\Omega_{t}\right)\right|^{-1}$ in the complex plane for $q=1.6$ and $q=3$, for various concentrations of fast ions. The solutions of the dispersion relation correspond to the high values of $D^{-1}\left(\Omega_{t}\right)$, which are recognizable as the fully filled circles. In particular, one can see that in the absence of fast particles, no circle is present in the upper part of the complex plane, where $\operatorname{Im}\left(\Omega_{t}\right)>0$ (which corresponds to excited modes). One zero of the dispersion relation is present slightly below the real axis: it is the GAM. Several zeros are present in the lower complex plane (where $\left.\operatorname{Im}\left(\Omega_{t}\right)<0\right)$ : those zeros correspond to highly damped modes, which are therefore observed neither in experiments nor in simulations. When fast particles are added, the positions of the roots evolve, and when the fast particle concentration becomes high enough, one of the roots crosses the real axis and finds itself in the upper plane.

On those figures, the EGAM is defined as the only mode which lies in the upper plane above a certain fast-particle density threshold, and which is therefore the only mode to be excited. During its evolution, this mode is labelled as an EGAM on figures 1 and 2. Another mode lies very close to the real axis above a certain fast particle density threshold, at a real frequency greater than that of the EGAM. However, this mode is never excited. The GAM is defined as the only mode which lies very close to the real axis in the absence of fast particles. When fast particles are added, it can either turn into an EGAM (figure 1) or remain on the real axis (figure 2) and thus not be excited.

Figure 4 was obtained with the same parameters as those used in Ref. 12: $q=3, T_{t} / T_{e}=1, \bar{u}_{\|}=2.8$, $\tau_{k}=1, m_{k} / m_{t}=1, Z_{k}=Z_{t}=1$ (NB: due to different normalizations, $\bar{\zeta}=4$ in Ref. 12 corresponds to $\bar{u}_{\|}=2.8$ in the present work). On that figure, we can see that the frequency of the EGAM is approximately half the frequency of the GAM which would be obtained in the absence of fast particles. For the real part of $\Omega_{t}$, we thus recover with a linear, analytical model the result which had previously been obtained in Ref. 12 with the non-linear, 5D gyrokinetic code GYSELA, and which had also been obtained experimentally in DIII-D (Ref. 7).

On figure 1, when the proportion of fast particles increases, the GAM becomes an EGAM: this mode is called an EGAM from GAM. At the same time, a mode which was deeply damped in the absence of fast particles progressively gets nearer to the real axis. The behaviour of the modes on figure 2 is different. When the proportion of fast particles increases, the GAM remains close to the real axis, and does not turn into an excited mode. At the same time, a mode which was deeply damped in the absence of fast particles progressively moves towards the upper plane, crosses the real axis and then gets excited: this mode is called a Landau EGAM. It is worth noting that the link between GAMs and EGAMs is thus different according to the value of the safety factor $q$ : for $q=1.6$, the EGAM originates from the GAM; while for $q=3$, the GAM and the EGAM belong to different mode branches. The existence of two different kinds of EGAMs has also been shown numerically ${ }^{21}$ with the gyrokinetic code $\mathrm{NEMORB}^{22,23}$.

On figures 3 and 4 are shown the contour representations (in blue) of $\left|D\left(\Omega_{t}\right)\right|^{-1}$ in the complex plane for $q=1.6$ and $q=3$, in the absence of fast particles. Those two contour representations are the same as the ones on figures 1 and 2 in the absence of fast particles. Atop those contour representations are superposed the evolutions of some zeros of the dispersion relation when the proportion of fast particles increases from 0 to $40 \%$ (black curves). The evolutions of those zeros are numerically computed; the evolutions of the other zeros are not represented as they are not significant. On figure 3, drawn with the same parameters as figure 1, one can see that the GAM becomes an EGAM (solid black line). At the same time, a mode which was deeply damped in the absence of fast 

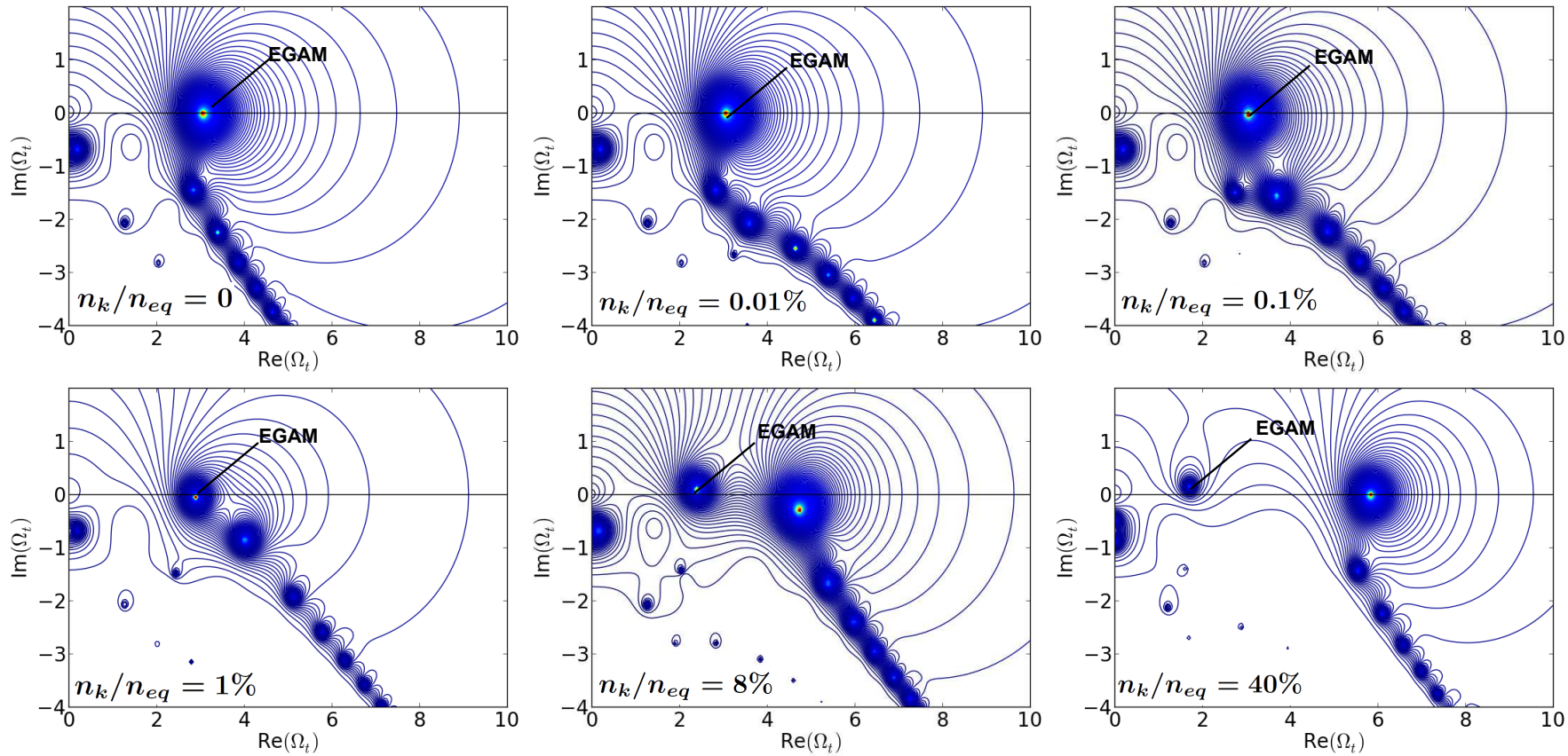

Figure 1. Contour representations of $\left|D\left(\Omega_{t}\right)\right|^{-1}$ in the complex plane for $q=1.6, T_{t} / T_{e}=1$, for various concentrations of fast ions, from 0 to $40 \%$. The bulk and fast ions belong to the same species (D-D), with $\bar{u}_{\|}=2.8$ and $\tau_{k}=1$.
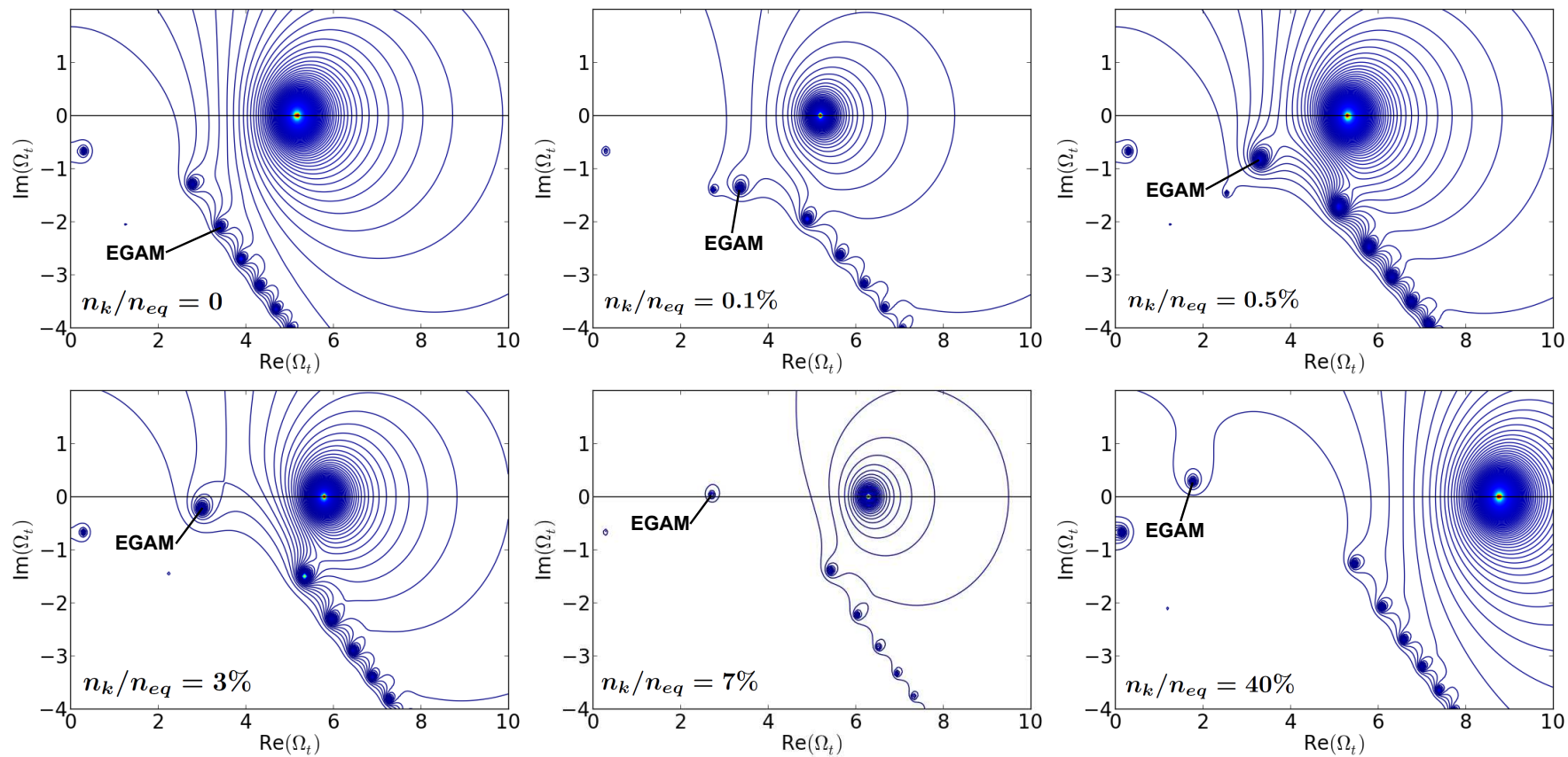

Figure 2. Contour representations of $\left|D\left(\Omega_{t}\right)\right|^{-1}$ in the complex plane for $q=3, T_{t} / T_{e}=1$, for various concentrations of fast ions, from 0 to $40 \%$. The bulk and fast ions belong to the same species (D-D), with $\bar{u}_{\|}=2.8$ and $\tau_{k}=1$.

particles gets very close to the real axis, but does not cross it (dashed black line). On figure 4, drawn with the same parameters as figure 2, one can see that the GAM does not turn into an EGAM: the GAM stays about the real axis, but never enters the upper plane (dashed black line). At the same time, a mode which was deeply damped in the absence of fast particles goes up, crosses the real axis, and yields an EGAM (solid black line).

\section{B. Impact of other parameters}

The safety factor $q$ is not the only parameter to have an impact on the link between GAMs and EGAMs. The 


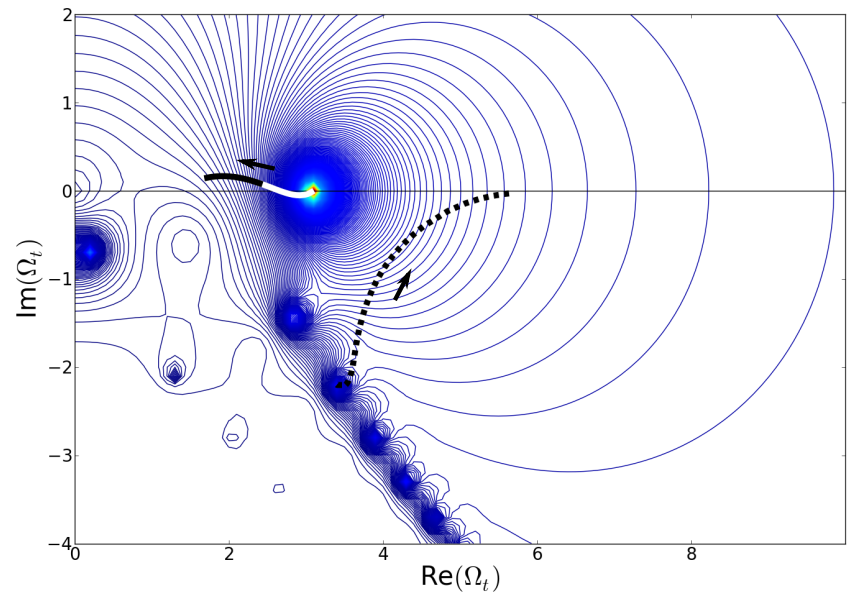

Figure 3. Background (blue curves): contour representation of $\left|D\left(\Omega_{t}\right)\right|^{-1}$ in the complex plane for $q=1.6, T_{t} / T_{e}=1$ and in the absence of fast ions. Black curves: evolution of the GAM and EGAM frequencies when the proportion of fast ions $n_{k} / n_{e q}$ varies from 0 to $40 \%$. The bulk and fast ions belong to the same species (D-D), with $\bar{u}_{\|}=2.8$ and $\tau_{k}=1$.

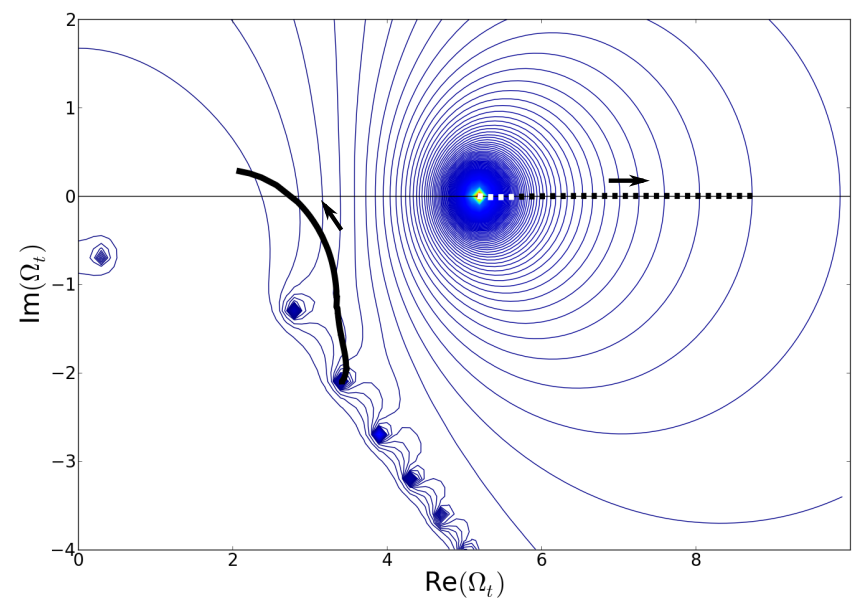

Figure 4. Background (blue curves): contour representation of $\left|D\left(\Omega_{t}\right)\right|^{-1}$ in the complex plane for $q=3, T_{t} / T_{e}=1$ and in the absence of fast ions. Black curves: evolution of the GAM and EGAM frequencies when the proportion of fast ions $n_{k} / n_{e q}$ varies from 0 to $40 \%$. The bulk and fast ions belong to the same species (D-D), with $\bar{u}_{\|}=2.8$ and $\tau_{k}=1$.

phase diagrams reproduced as figures 5, 6 and 7 show, for various sets of parameters, three different zones in which the EGAM either originates from the GAM, is a Laudau EGAM, or is not excited at all (at least up to a fast particle fraction of $40 \%)$. Those sets of parameters are respectively $\left(q, \bar{u}_{\|}\right),\left(q, T_{t} / T_{e}\right)$ and $\left(q, \tau_{k}\right)$. In the zones labelled as EGAM from GAM, the EGAM is excited and originates from the GAM: the situation corresponds to the one which appears on figures 1 and 3. In the zones labelled as Landau EGAM, the EGAM is excited and originates from a mode which is deeply damped in the absence of fast particles: the situation corresponds to the one which appears on figures 2 and 4 .

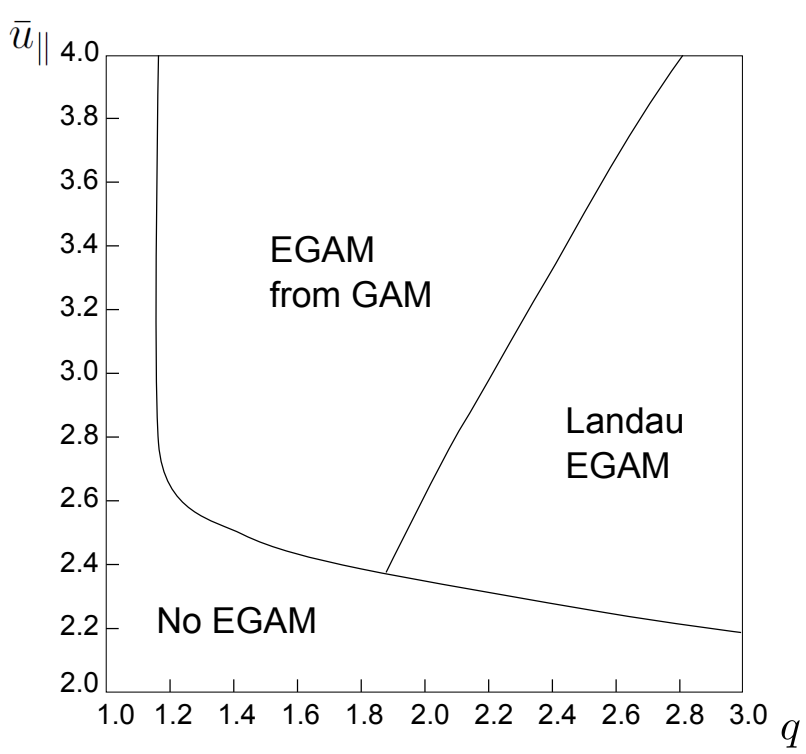

Figure 5. Phase diagram: type of EGAM as a function of $q$ and $\bar{u}_{\|}$, for D-D particles, with $T_{t} / T_{e}=1$ and $\tau_{k}=1$.

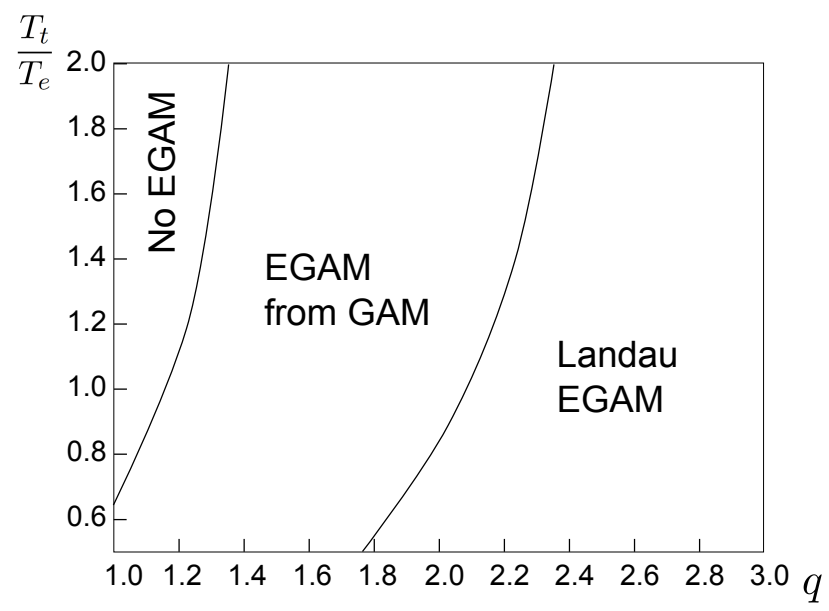

Figure 6. Phase diagram: type of EGAM as a function of $q$ and $T_{t} / T_{e}$, for D-D particles, with $\bar{u}_{\|}=2.8$ and $\tau_{k}=1$.

Figure 8 completes figure 7: the three different zones No EGAM, EGAM from GAM and Landau EGAM are represented as functions of $q$ and $\tau_{k}$ for two different kinds of fast particles, which have the same charge but different masses (Hydrogen and Deuterium). The thermal particles are Deuterium in both cases.

Finally, figure 9 gives further information about the impact of the charge number. On this figure, the same three zones are represented as functions of $q$ and $\tau_{k}$ for two different kinds of fast particles, which have the same mass but different charges (Tritium and Helium 3). The thermal particles are Deuterium in both cases.

It is to be noted that in figures 5 to 9 , the critical density above which the EGAM becomes unstable is dif- 


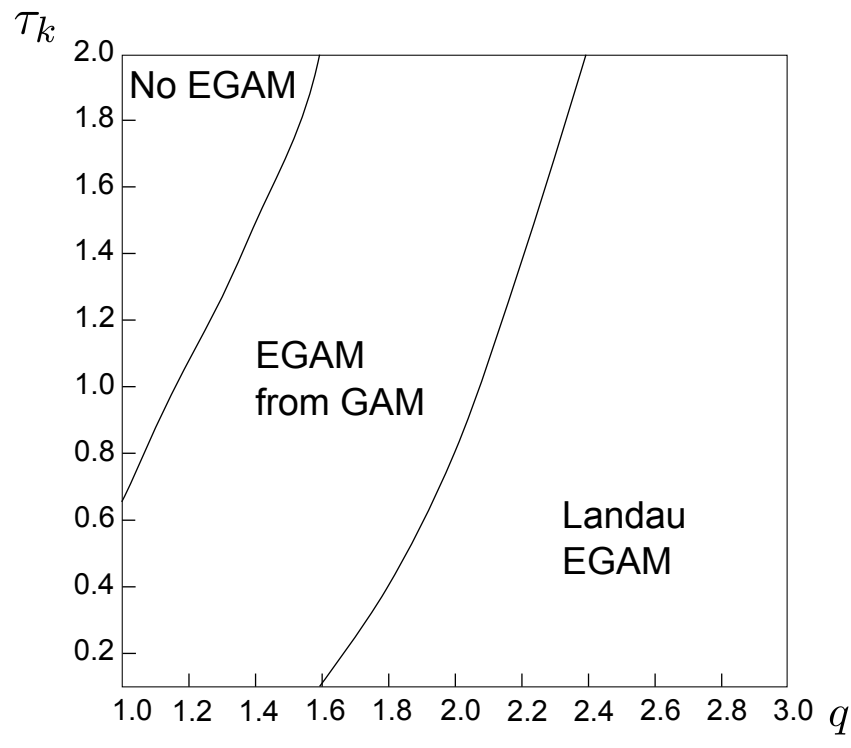

Figure 7. Phase diagram: type of EGAM as a function of $q$ and $\tau_{k}$, for D-D particles, with $\bar{u}_{\|}=2.8$ and $T_{t} / T_{e}=1$.

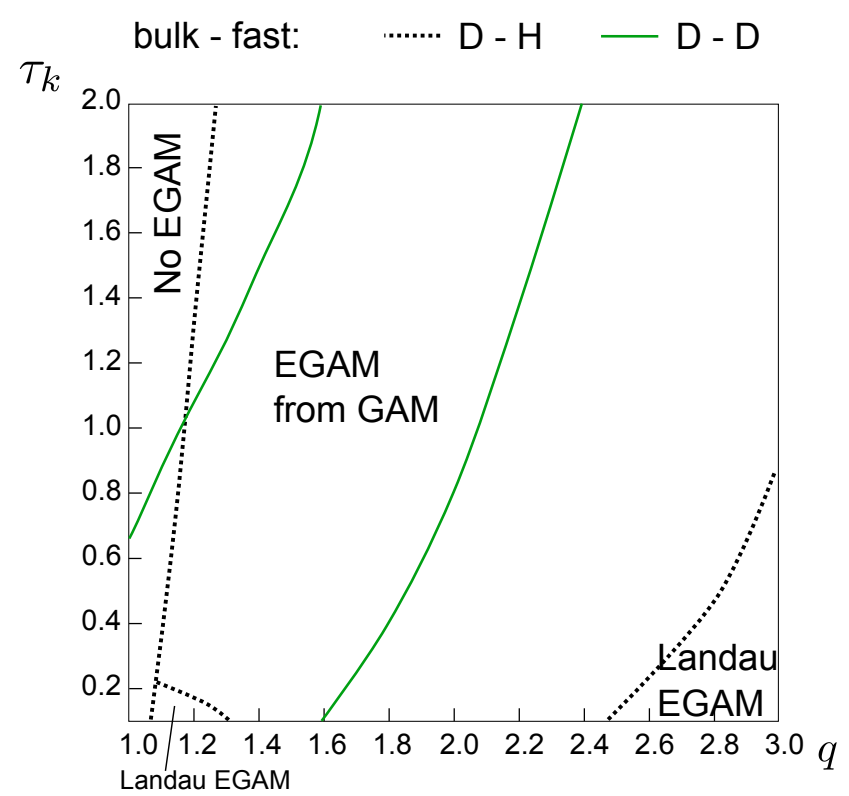

Figure 8. Phase diagram: type of EGAM as a function of $q$ and $\tau_{k}$, with $\bar{u}_{\|}=2.8$ and $T_{t} / T_{e}=1$, for $\mathrm{D}$ (bulk) $-\mathrm{H}$ (fast) and D-D particles.

ferent at each position in the diagram. Indeed, as noted in Ref. 12 , the excitation threshold depends on a certain number of parameters, including $q, \bar{u}_{\|}, \tau_{k}$ and $T_{t} / T_{e}$; this issue is further discussed in section III C. The No $E G A M$ region refers to cases in which no EGAM is excited when the fraction of energetic particles varies from 0 to $40 \%$; an EGAM is considered to be excited when $\operatorname{Im}\left(\Omega_{t}\right)$ is positive, with a precision of 0.05 . It is to be noted that diagrams 5 to 9 were obtained with a matrix of test points, with steps of 0.2 (for all parameters)

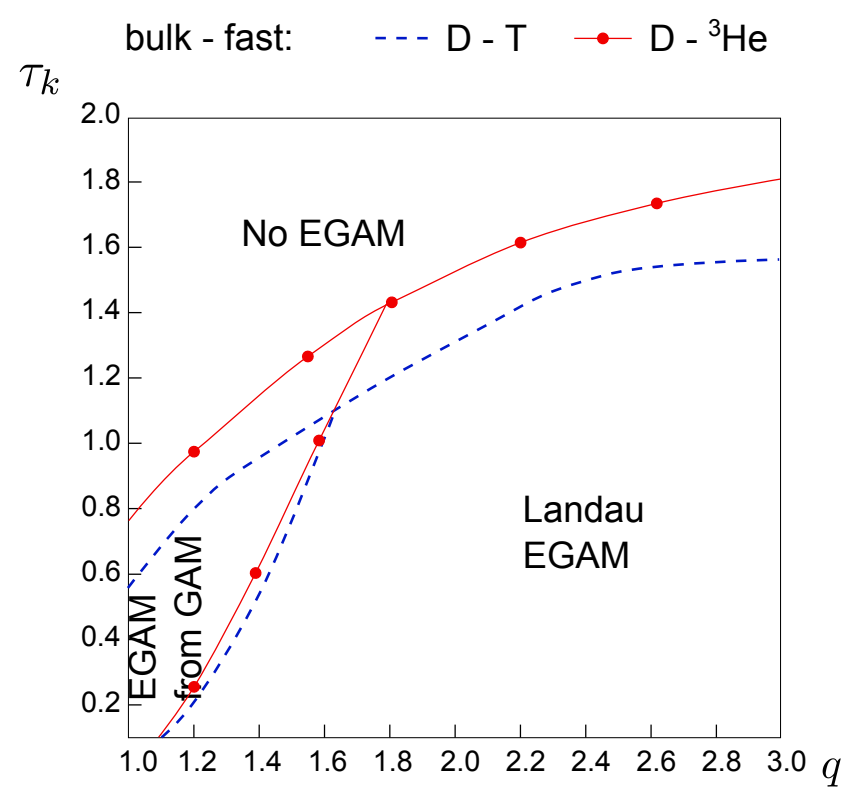

Figure 9. Phase diagram: type of EGAM as a function of $q$ and $\tau_{k}$, with $\bar{u}_{\|}=2.8$ and $T_{t} / T_{e}=1$, for $\mathrm{D}$ (bulk) - $\mathrm{T}$ (fast) and $\mathrm{D}$ (bulk) $-{ }^{3} \mathrm{He}$ (fast) particles.

between the points. For each test point, the dispersion relation 9 was numerically solved for different values of the fast particle density (from 0 to $40 \%$ ).

\section{Excitation threshold}

The parameters of the bulk plasma and of the fast particles also have an impact on the excitation threshold of EGAMs. Figure 10 presents the growth rate of EGAMs in the case of $q=1.8$ and Deuterium as thermal particles, for different species of fast particles $(\mathrm{H}, \mathrm{D}, \mathrm{T}$, and $\left.{ }^{3} \mathrm{He}\right)$. For the considered parameters, figure 8 shows that Hydrogen and Deuterium EGAMs originate from GAMs, while figure 9 shows that Tritium and Helium 3 EGAMs are Landau EGAMs. This distinction is consistent with what can be observed on figure 10: in the case of Hydrogen and Deuterium, we have $\lim _{n_{k} \rightarrow 0} \operatorname{Im}\left(\Omega_{t}\right) \approx 0$, which indicates that the EGAMs originate from GAMs. On the contrary, in the case of Tritium and Helium 3, we have $\lim _{n_{k} \rightarrow 0} \operatorname{Im}\left(\Omega_{t}\right)<0$, which indicates that the EGAMs are Landau EGAMs. Such a difference in the excitation threshold between the two types of EGAMs should allow one to distinguish a Landau EGAM from an EGAM from GAM in experiments.

With the parameters used to produce figure 10, for an experimentally reasonable density of fast particles of $5 \%$, we can see that there is a huge difference between the different species. For heavy fast particles such as Tritium and Helium 3, the EGAMs (which are then Landau EGAMs) are still far from the excitation threshold. On the contrary, for lighter fast particles such as Deuterium 


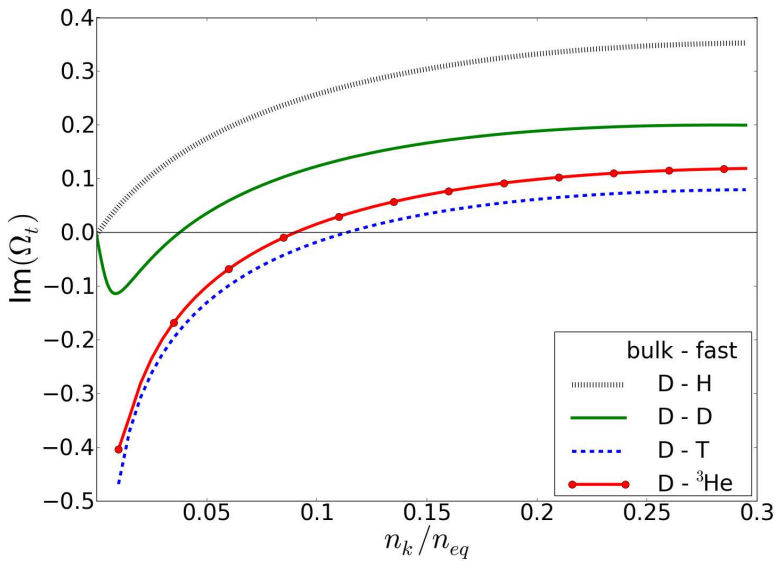

Figure 10. Normalised EGAM growth rate $\operatorname{Im}(\Omega)$ as a function of $n_{k} / n_{e q}$, with $q=1.8, \bar{u}_{\|}=2.8 \tau_{k}=1$ and $T_{t} / T_{e}=1$, for $\mathrm{D}$ (bulk) - $\mathrm{H}$ (fast), D-D, D(bulk) - T(fast) and $\mathrm{D}$ (bulk) $-{ }^{3} \mathrm{He}$ (fast) particles. The EGAM is excited whenever $\operatorname{Im}(\Omega)>0$.

and especially Hydrogen, a density of $5 \%$ corresponds to a region where the EGAMs (which are then EGAMs from GAMs) can be excited.

In addition, it is to be noted that there is no fast particle density threshold for the excitation of EGAMs in the Hydrogen case, for the parameters used to compute figure 10. It means that there exist certain cases in which EGAMs are very easily excited. This feature is related to the nature of the EGAM (the excitation is easier in the case of an EGAM from GAM). The behaviour of EGAMs at very low fast particle concentration is of notable interest for the study of the interaction of EGAMs with turbulence, and to understand the apparition of EGAMs in experiments.

\section{DISCUSSION}

\section{A. Limit $q \rightarrow 0$}

When $q \rightarrow 0$, figures 5 to 9 show that no EGAM is excited. Let us show that it is consistent with expression 9. First, let us show that, in the absence of fast particles , $\left|\Omega_{t, G A M}\right|$ remains small (under a few unities) when $q \rightarrow 0$. Indeed, if $\left|\Omega_{t, G A M}\right|$ were large, the hydrodynamic limit would be applicable, and the dispersion relation would read in that $\operatorname{limit}^{19}$ : $1-\frac{q^{2}}{\Omega_{t}^{2}}\left(\frac{7}{4}+\frac{T_{e}}{T_{t}}\right)+i \sqrt{\pi} q^{2} \Omega_{t}^{3} e^{-\Omega_{t}^{2}}=0$. Taking into account the constraint on $\Omega_{t}$, which has to be above a few unities for the hydrodynamic limit to be valid, this equation has no solution (in $\Omega_{t}$ ) when $q \rightarrow 0$. Therefore, the only possibility for $\Omega_{t, G A M}$ to be a solution of the dispersion relation (7) is to be out of the hydrodynamic limit, that is to say to be smaller than a few unities. Let us now consider the EGAM frequency $\Omega_{t, E G A M}$ : it is expected to have the same order of magnitude as $\Omega_{t, G A M}$, even though there may be a ratio of several unities between the two frequencies. When $q$ approaches $0, \Omega_{t, E G A M}$ is therefore to be looked for in a region of the type $\left|\Omega_{t}\right|<C$, where $C$ is an arbitrary, positive constant of the order of a few unities. It can be checked with a numerical graphic representation of $D_{1}\left(\Omega_{t}\right)+D_{2}\left(\Omega_{k}\right)$ that this expression does not have any root satisfying $\left|\Omega_{t}\right|<C, \operatorname{Re}\left(\Omega_{t}\right)>0$ and $\operatorname{Im}\left(\Omega_{t}\right)>0$; which means that $1 /\left(D_{1}\left(\Omega_{t}\right)+D_{2}\left(\Omega_{k}\right)\right)$ is bounded in that region (called $U$ from now on). In addition, since the plasma function $\mathcal{Z}(\Omega)$ is bounded for $\operatorname{Im}(\Omega)>C^{\prime}$ (where $C^{\prime}$ can be any arbitrary, positive or negative constant), $A_{1}\left(\Omega_{t}\right)+A_{2}\left(\Omega_{k}\right)-\frac{\left[N_{1}\left(\Omega_{t}\right)+N_{2}\left(\Omega_{k}\right)\right]^{2}}{D_{1}\left(\Omega_{t}\right)+D_{2}\left(\Omega_{k}\right)}$ is bounded for $\Omega_{t}$ in $U$. It is important to note that this last expression does not have any explicit dependency in $q$, which implies that it remains bounded even when $q$ approaches 0 . Let us now consider the remainder of the dispersion relation: $\lim _{q \rightarrow 0} \frac{1}{q^{2}}=+\infty$. Therefore, when $q \rightarrow 0$, one part of the dispersion relation approaches infinity, while the other remains bounded for values of $\Omega_{t}$ in $U$ : consequently, the dispersion relation (9) does not have any excited solution when $q \rightarrow 0$, which is consistent with what can be observed on figures 5 to 9 .

\section{B. Limits $\bar{u}_{\|} \rightarrow 0$ and $\tau_{k} \rightarrow+\infty$}

It can be observed on figure 5 that when $\bar{u}_{\|} \rightarrow 0$, no EGAM is excited. To account for this phenomenon, it is to be remembered that the growth of the EGAM comes from the existence of a range of energy for which ${ }^{9,12}$ $\frac{\partial f_{s}}{\partial \varepsilon_{s}}>0$. When $\bar{u}_{\|} \rightarrow 0$, the centres of the fast ion bumps move towards 0 in the phase space; the overall ion distribution function thus approaches a Maxwellian, for which $\frac{\partial f_{s}}{\partial \varepsilon_{s}}>0$ is nowhere verified. Consequently, no EGAM is expected to be excited, which is consistent with the observation on figure 5. For the same reason, no EGAM is expected to be excited when $\tau_{k} \rightarrow+\infty$, which is consistent with figures 7 to 9 .

\section{Impact of FLR/FOW effects on the growth rate}

On figure 10, in the $\mathrm{D}-\mathrm{D}$ case, the growth rate becomes positive for $n_{k} / n_{e q}=4 \%( \pm 0.5$ percentage point). In the case of $q=3$, we find that the growth rate of EGAMs in the D-D case, (with $\bar{u}_{\|}=2.8, \tau_{k}=1$ and $T_{t} / T_{e}=1$ ) becomes positive for $n_{k} / n_{e q}=6 \%$. This value is quite low when compared with the excitation threshold obtained with the code GYSELA for the same parameters ${ }^{12}$ : the threshold value was then about $n_{k} / n_{e q}=15 \%$. A possible explanation for this discrepancy is that GYSELA takes into account the Finite Larmor Radius (FLR) and Finite Orbit Width (FOW) 
effects, while the present model does not. Regarding the FOW effects, a numerical plot in Ref. 24, based on a theoretical model from Ref. 25, 26 shows that for large values of $q(q>2)$, the damping rate of GAMs in the absence of fast particles is several orders of magnitude larger when FOW effects are taken into account than when they are not. Consequently, for large values of $q$, the EGAM growth rate is expected to be lower when FOW effects are taken into account than when they are not. In addition, regarding the FLR effects, the GYSELA simulations ${ }^{12}$ were performed over an annular domain $0.2 \leq r / a \leq 0.8$, where $r$ is the radial coordinate, and $a$ is the maximal value of $r$. This restricted choice of $r / a$ led to higher values of the radial wave vector of the mode $k_{r}$, and therefore artificially increased the damping due to FLR effects. Both those FOW and FLR effects are consistent with the difference found in threshold values between the present analytical model and the GYSELA simulations ${ }^{12}$.

It is to be noted that according to the same numerical plot ${ }^{24}$, the FOW effects are expected to play a less significant role for low values of $q(q \leq 2)$. As a matter of fact, simulations taking FOW effects into account made with the gyrokinetic code NEMORB, in the DD case with parameters $q=2, \bar{u}_{\|}=2.8, \tau_{k}=1$ and $T_{t} / T_{e}=1$, indicate ${ }^{21}$ that the EGAM excitation threshold is reached at $n_{k} / n_{e q}=5 \%$. With the present analytical model, we find for the same parameters a threshold of $n_{k} / n_{e q}=4 \%$. Contrary to what was found in the $q=3$ case, the threshold values found in the $q=2$ case are similar whether FOW effects are taken into account (Ref. 21) or not (present model). This is consistent with Ref. 24. In that NEMORB case, the simulations were performed over a domain $0 \leq r / a \leq 1$, thus leading to reduced FLR effects with respect to the GYSELA case.

Further details about FOW/FLR effects and numerical analysis of EGAM excitation can be found in Ref. 21.

\section{EGAM resonance}

The resonance in the model happens for $\Omega_{k}=\bar{u}_{\|}$. When $\bar{u}_{\|}$and $\Omega_{k, G A M}$ have similar values, it is expected that the fast particles will preferentially excite the GAM mode (which is the least damped mode in the absence of fast particles), thus leading to an EGAM from GAM. In the absence of fast particles, when $q$ increases, $\Omega_{k, G A M}$ also increases ${ }^{19}$, while numerical plots made in the present work indicate that the damped modes do not evolve significantly. If $q$ increases from a configuration in which $\bar{u}_{\|}$and $\Omega_{k, G A M}$ have similar values (while $\bar{u}_{\|}$ remains the same), $\Omega_{k, G A M}$ will increase and thus move away from $\bar{u}_{\|}$. At the same time, the damped modes will keep the same values, one of them having a real part close to $\bar{u}_{\|}$. The fast particles will then resonate with this damped mode and excite it, rather than the GAM which has moved away, thus leading to a Landau

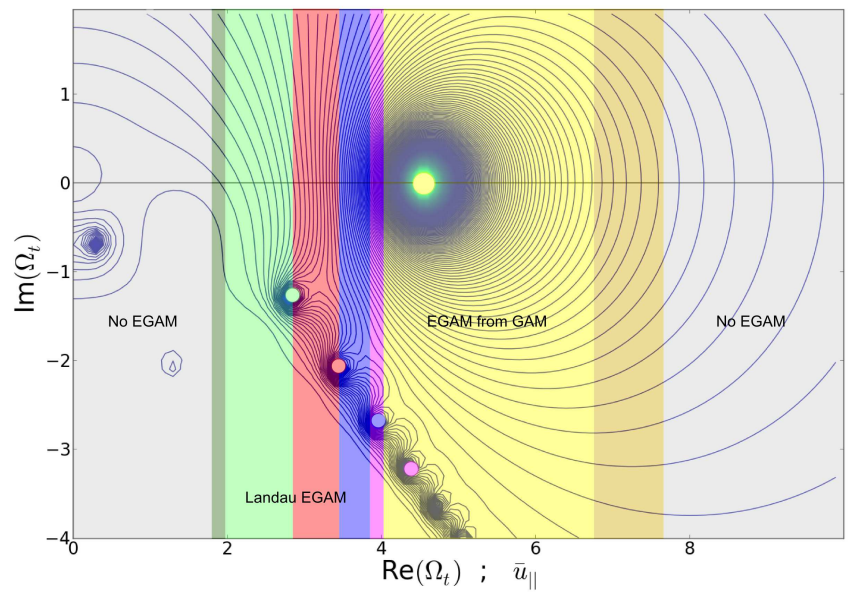

Figure 11. Background: contour representation of $\left|D\left(\Omega_{t}\right)\right|^{-1}$ in the complex plane for $q=2.6, T_{t} / T_{e}=1$ and in the absence of fast ions. Coloured stripes: $\bar{u}_{\|}$. This figure allows to see the resonance link between $\bar{u}_{\|}$(coloured stripes) and the roots of the GAM dispersion relation in the absence of fast particles (coloured circles), when bulk and fast ions belong to the same species (D-D) and when $\tau_{k}=0.4$. Reading example: for a value of $2.5, \bar{u}_{\|}$is in the light green stripe. This means that it is the root tagged with a light green circle that will be excited and turn into the EGAM when fast particles are added.

EGAM. Consistently, still starting from a configuration in which $\bar{u}_{\|}$and $\Omega_{k, G A M}$ have similar values, if $\bar{u}_{\|}$ decreases while $q$ remains the same, $\bar{u}_{\|}$will move away from $\Omega_{k, G A M}$ : the fast particles are therefore expected to excite a damped mode with a real part close to $\bar{u}_{\|}$ rather than the GAM, thus leading to a Landau EGAM. This analysis is consistent with what can be observed on figure 5: when $q$ increases (for a given $\bar{u}_{\|}$) or when $\bar{u}_{\|}$ decreases (for a given $q$ ), there is a transition from the $E G A M$ from $G A M$ zone to the Landau EGAM zone. The almost straight-line limit between those two zones was also expected, as it reflects the role of $\bar{u}_{\|} / q$ in the resonance $\omega_{G A M}=\sqrt{2 T_{t} / m_{k}}\left(\bar{u}_{\|} / q R\right)$ (expressed in terms of the non-normalized frequency $\omega_{G A M}$ ).

Figure 11 (on which bulk and fast particles belong to the same species, which means that $\Omega_{k}=\Omega_{t}$ ) gives a clear illustration of the resonance $\Omega_{k}=\bar{u}_{\|}$when $q=2.6$ is fixed and $\bar{u}_{\|}$varies, with $\tau_{k}=0.4$. It can be seen that the fast particles always excite a root of the dispersion relation which has a real part $\operatorname{Re}\left(\Omega_{t}\right)$ very close to $\bar{u}_{\|}$; in fact it is the first mode which has a real frequency superior to $\bar{u}_{\|}$which is excited - with a preference for the GAM mode when two modes have similar real frequencies, given that this mode is already very close to the excitation threshold, and therefore requires less energy to be excited than the highly damped modes.

Note that below a certain value of $\bar{u}_{\|}\left(\bar{u}_{\|}=1.8\right.$ on figure 11), the EGAMs are not excited - we here remind that the EGAM is considered not to be excited when it never has a positive growth rate for fractions of fast 
particles ranging between 0 and $40 \%$, with a precision of 0.05 for the growth rate $\operatorname{Im}\left(\Omega_{t}\right)$. For $\bar{u}_{\|}$between 1.8 and 2 (in dark green on figure 11), the EGAM is a Landau EGAM: it corresponds to a root of the dispersion relation which does not exist in the absence of fast particles, and which appears close to the root tagged with a light green circle when fast particles are added. For $\bar{u}_{\|}$between 2 and 4, the Landau EGAMs come from roots which all exist in the absence of fast particles, and which are tagged with colour circles on figure 11. For $\bar{u}_{\|}$between 4 and 6.7, the EGAM comes from the GAM root (in light yellow on figure 11).

Beyond a certain value of $\bar{u}_{\|}\left(\bar{u}_{\|}=7.6\right.$ on figure 11), the EGAMs are no more excited. $\bar{u}_{\|}$is too far from the GAM frequency for a resonance to occur, and the other modes are too deeply damped. Between the GAM from $E G A M$ range and the no-EGAM range, there is a zone (in dark yellow on figure 11) where the origin of the EGAM remains unclear. The reason is that when fast particles are added, a deeply damped mode first moves to the real axis and joins the GAM root: those two modes then become undistinguishable. When the fraction of fast particles further increases, one of them turns into the EGAM while the other remains below the real axis.

When $\tau_{k}$ increases, the bumps of the fast-particle distribution function become larger, and consequently the possibilities of resonance between $\Omega_{k, G A M}$ and $\bar{u}_{\|}$increase. When such possibilities of resonance increase, a resonance with the GAM is preferred to a resonance with a damped mode, since the GAM is already much closer to the excitation threshold. This can be observed on figures 7 and 8: when $\tau_{k}$ increases (for given values of $q$ and $\left.\bar{u}_{\|}\right)$, there is a transition from the Landau EGAM zone to the EGAM from GAM zone.

\section{CONCLUSION}

The ratio of frequencies between the EGAM and the GAM close to $1 / 2$ observed in experiments in DIII-D ${ }^{7}$ and in the non-linear code GYSELA ${ }^{12}$ is recovered with a linear model. A noticeable feature of this linear model is that quantitative results can be obtained in a few minutes through numerical computation on a personal computer. This model however exhibits some limitations. First, kinetic electrons are not taken into account, while they might play a role in the excitation of EGAMs, similarly to the effect observed for standard $\mathrm{GAMs}^{27}$. Second, trapped ions are not considered either. Finally, neither FLR nor FOW effects are taken into account. The impacts of FLR/FOW effects on EGAMs, together with a detailed comparison of the analytic dispersion relation with gyrokinetic simulations, are analysed in Ref. 21.

Depending on the equilibrium parameters of the plasma, as well as on the parameters of the energetic particles, the GAMs and EGAMs belong or not to the same branch. The EGAM originates from the GAM in the following regions of the studied parameters: low values of the safety factor $q$ and of the mass of the fast ions; high values of the energy of the fast ions, of the width of the fast particle distribution, and of the ratio of the bulk ion temperature to the electron temperature. On the contrary, the EGAM originates from a mode which is damped in the absence of fast particles (Landau EGAM) when the studied parameters are in the following regions: high values of the safety factor $q$ and of the mass of the fast ions; low values of the energy of the fast ions, of the width of the fast particle distribution, and of the ratio of the bulk ion temperature to the electron temperature.

The difference between EGAMs originating from GAMs and Landau EGAMs may be observed in experiments through the different density thresholds required for their excitation (figure 10). Information on the behaviour of EGAMs for low concentrations of fast particles can be retrieved from the present model and may be compared to experimental results. It may also be possible to differentiate the two types of EGAM through their radial profiles: since they have different origins, an EGAM from GAM and a Landau EGAM may have different radial structure. If this happened to be the case, it would then be possible to observe it in experiments. Clarification on the EGAM radial structure and the potential differences between different kinds of EGAMs requires additional theoretical and experimental work.

Differentiating the GAM from the EGAM in experiments has several interests. Contrary to the GAM, which arises from turbulence, the EGAM can give information on the fast particle distribution. For instance, the nature of the EGAM (EGAM from GAM or Landau EGAM) can give information on the mean velocity of the fast particles (parameter $\bar{u}_{\|}$on figure 9). Besides, if it is found from further investigation that the EGAMs have different radial structures depending on their nature, their impact on turbulence may prove different as well. Since the study of EGAMs is largely motivated by their impact on turbulence, it is worth trying to understand whether different types of interaction with turbulence may exist, depending on the EGAM type.

\section{ACKNOWLEDGMENTS}

This work was part-funded by the RCUK Energy Programme [grant number EP/I501045] and the European Union's Horizon 2020 research and innovation programme [Enabling Research Project CfP-WP14-ER01/ENEA Frascati-01]. The views and opinions expressed herein do not necessarily reflect those of the European Commission.

The authors would like to thank Boris Breizman, Michael Fitzgerald, Matthew Lilley, Timothée Nicolas and Fulvio Zonca for fruitful discussion. One of the authors (D. Zarzoso) kindly acknowledges seminal discussions with A. Smolyakov about the nature of the EGAM 
and its existence in the limit of no energetic particles.

\section{APPENDIX}

In this appendix are presented the major steps of the derivation of the EGAM dispersion relation (9). The model takes into account three species: two species of ions (thermal ions and kinetic ions), together with the electrons. Throughout the calculus, the subscripts $t, k$ and $e$ are used to denote respectively the thermal ions, the kinetic ions and the electrons. Whenever an equation is the same for thermal and kinetic ions, it is written only once for simplicity purpose, and the subscript $s$ (standing for species) is used. This means that whenever the subscript $s$ is met in an equation, it can be substituted indifferently with $t$ or $k$.

The quasi-neutrality equation is solved taking into account the three species. This equation is coupled to two gyrokinetic equations for the ions, while an adiabatic representation is used for the electrons.

\section{A. Gyrokinetic equation, for thermal and kinetic ions}

The equation used for species $s$ (where it is recalled that $s$ stands indifferently for $t$ - thermal - or $k$ - kinetic) is the gyrokinetic equation (1). We recall that the distribution function of the ions of species $s$ is decomposed into an equilibrium part $F_{s}$ and a perturbed part $\delta f_{s}$ :

$$
f_{s}=F_{s}\left(1+\delta f_{s}\right)
$$

The model is electrostatic: no fluctuation of the magnetic field is accounted for.

The thermal ion equilibrium is described by a Maxwellian distribution function (expression (6)). The temperature and density equilibrium radial profiles are assumed to be flat, which means that $F_{t}$ depends on the kinetic energy of the particles $\varepsilon_{t}$ only.

The kinetic ion equilibrium is described by a so-called bump-on-tail function, consisting in two Maxwellian functions shifted in parallel velocity (expression (8)).

Note that the fast particles are accelerated in the parallel direction only: the fast particle parallel velocity is of the order of a few times the thermal velocity, while their perpendicular velocity is of the order of the thermal velocity itself. In addition, the aspect ratio $R_{0} / a$ is assumed to be large. Consequently, the vast majority of the fast particles here considered are highly passing. Their parallel velocity can therefore be considered as a motion invariant, $\bar{v}_{\|}$can be chosen to be a constant (i.e. independent of $r$ and $\theta$ ), and the distribution function (8) can be recast as a function of motion invariants only:

$$
F_{k}=n_{k}\left(\frac{m_{k}}{2 \pi T_{t} \tau_{k}}\right)^{\frac{3}{2}} e^{-\frac{\frac{1}{2} m_{k} \bar{v}_{\|}^{2}+\varepsilon_{k}}{T_{t} \tau_{k}}} \cosh \left(\frac{m_{k} v_{\|} \bar{v}_{\|}}{T_{t} \tau_{k}}\right)
$$

Two different kinds of expansions are performed: a) the equations are linearised according to the perturbed quantities $\left(\delta f_{t}, \delta f_{k}\right.$ and $\left.\phi\right)$;

b) the equations are expanded up to the first order in $\rho_{*}$, consistently with the order at which the gyrokinetic equations used in the model are valid. $\rho_{*}$ is defined as $\rho_{i} / a$ where $\rho_{c}$ is the ion Larmor radius and $a$ the minor radius of the tokamak. Note that this expansion is valid for both thermal and kinetic ions: kinetic ions are accelerated only in the parallel direction, which means that $\rho_{*}$ is the same for thermal and kinetic ions.

After linearisation according to the perturbed quantities, the gyrokinetic equation for species $s$ reads:

$$
F_{s} \partial_{t} \delta f_{s}+\mathbf{v}_{E} \cdot \nabla_{\perp} F_{s}+F_{s} \mathbf{v}_{g, s} \cdot \nabla_{\perp} \delta f_{s}+F_{s} v_{\|} \nabla_{\|} \delta f_{s}+d_{t} \varepsilon_{s} \partial_{\varepsilon_{s}} F_{s}=0
$$

Neither $F_{t}$ nor $F_{k}$ have any spatial dependency: consequently $\nabla_{\perp} F_{s}=0$.

Besides, the kinetic energy of species $s$ varies according to the electric energy transfers: $d_{t} \varepsilon_{s}=-e_{s} v_{\|} \nabla_{\|} \phi-$ $e_{s} \mathbf{v}_{g, s} \cdot \nabla_{\perp} \phi$. Consequently, the gyrokinetic equation for species $s$ can be recast as:

$$
\partial_{t} \delta f_{s}=\left(\mathbf{v}_{g, s} \cdot \nabla_{\perp}+v_{\|} \nabla_{\|}\right)\left(\phi Z_{s} T_{t} \partial_{\varepsilon_{s}} \log F_{s}-\delta f_{s}\right)
$$

where $Z_{s}=e_{s} / e$ is the charge number of species $s, e$ is the elementary Coulomb charge, and $\phi$ has been normalized to $T_{t} / e$.

Since the GAM and the EGAM are axisymmetric modes, the perturbed quantities $\delta f_{t}, \delta f_{k}$ and $\phi$ are independent of the toroidal angle $\varphi$. In the case of a tokamak with circular, concentric magnetic flux surfaces, we have, in flux coordinates:

$$
\begin{aligned}
\mathbf{v}_{g, s} \cdot \nabla_{\perp} & =v_{g, s}\left(\sin \theta \partial_{r}-\frac{\cos \theta}{r} \partial_{\theta}\right) \\
v_{\|} \nabla_{\|} & =\frac{v_{\|}}{q R} \partial_{\theta}
\end{aligned}
$$

where $v_{g, s}=\frac{m_{s} v_{\|}^{2}+\mu_{s} B}{e_{s} R B}$.

The radial extension of the EGAM is assumed to be significantly smaller than the minor radius of the tokamak. Therefore, in expression (14), only the $\sin \theta \partial_{r}$ term 
is retained as it is assumed to be dominant over the $\frac{\cos \theta}{r} \partial_{\theta}$ term.

A decomposition of $\delta f_{t}, \delta f_{k}$ and $\phi$ is performed on a Fourier basis:

$$
\delta f_{s}=\sum_{\substack{\omega, m=0 \\ k, m=-\infty}}^{+\infty}\left[\delta f_{s m}^{c} \cos m \theta+\delta f_{s m}^{s} \sin m \theta\right] e^{i\left(k_{r} r-\omega t\right)}
$$

$$
\phi=\sum_{\substack{\omega, m=0 \\ k_{r}=-\infty}}^{+\infty}\left[\phi_{m}^{c} \cos m \theta+\phi_{m}^{s} \sin m \theta\right] e^{i\left(k_{r} r-\omega t\right)}
$$

At leading order in $\rho_{*}$, the first two poloidal harmonics of equation (13) read:

$$
\begin{aligned}
2 \omega \delta f_{s 0} & =v_{g, s} k_{r}\left[\delta f_{s 1}^{s}-\phi_{1}^{s} Z_{s} T_{t} \partial_{\varepsilon_{s}} \log F_{s}\right] \\
i \omega \delta f_{s 1}^{c} & =\frac{v_{\|}}{q R}\left[\delta f_{s 1}^{s}-\phi_{1}^{s} Z_{s} T_{t} \partial_{\varepsilon_{s}} \log F_{s}\right] \\
i \omega \delta f_{s 1}^{s} & =-i \phi_{0} v_{g, s} k_{r} Z_{s} T_{t} \partial_{\varepsilon_{s}} \log F_{s}-\frac{v_{\|}}{q R}\left[\delta f_{s 1}^{c}-\phi_{1}^{c} Z_{s} T_{t} \partial_{\varepsilon_{s}} \log F_{s}\right]
\end{aligned}
$$

Note that those harmonics have been expressed up to the first order in $\rho_{*}$ only. To get them, $k_{r} \rho_{c}$ has been assumed to be of order $\rho_{*}^{1 / 2}$, leading to the following orderings (which can easily be checked a posteriori): $v_{\|} / q R \omega$ is of order 0 in $\rho_{*} ; \phi_{1}^{s} / \phi_{0}, \delta f_{s 1}^{s} / \phi_{0}, \delta f_{s 1}^{c} / \phi_{0}$ and $v_{g, s} k_{r} / \omega$ are of order $1 / 2$ in $\rho_{*} ; \delta f_{s 0} / \phi_{0}$ and $\phi_{1}^{c} / \phi_{0}$ are of order 1 in $\rho_{*}$. While $\delta f_{s 1}^{c} / \phi_{0}$ is of order $1 / 2$ in $\rho_{*}$, it is important to remark that its integral in velocity at that order is null, which means that $\delta n_{s 1}^{c} / \phi_{0}$ is of order 1 in $\rho_{*}$.

Defining $\omega_{g, s}=v_{g, s} k_{r}$ and $\omega_{\|}=v_{\|} / q R$, equations (18) to $(20)$ can, after some linear combinations, be recast as follows:

$$
\begin{aligned}
\delta f_{s 0} & =-Z_{s} T_{t} \partial_{\varepsilon_{s}} \log F_{s} \frac{\omega^{2} \omega_{g, s} \phi_{1}^{s}+\omega \omega_{g, s}^{2} \phi_{0}}{2 \omega\left(\omega^{2}-\omega_{\|}^{2}\right)} \\
\delta f_{s 1}^{s} & =-Z_{s} T_{t} \partial_{\varepsilon_{s}} \log F_{s} \frac{\omega \omega_{g, s} \phi_{0}+\omega_{\|}^{2} \phi_{1}^{s}+i \omega \omega_{\|} \phi_{1}^{c}}{\omega^{2}-\omega_{\|}^{2}}
\end{aligned}
$$

Equation (19) has been used to derive equations (21) and (22), but it will not be useful any more in what follows: it has therefore not been copied again. It is to be recalled that equations (21) and (22) are valid indifferently for thermal and kinetic ions (just replace the subscript $s$ with $t$ or $k$ ).

\section{B. Quasi-neutrality equation, taking into account thermal ions, kinetic ions and adiabatic electrons}

The perturbed quasi-neutrality equation reads: $\delta n_{e}=$ $Z_{t} \delta n_{t}+Z_{k} \delta n_{k}$.

The perturbed density of ion species $s$ reads, in the gyrokinetic framework at first order in $\rho_{*}$, as the integral of the perturbed gyro-centre distribution function, plus a polarisation term:

$$
\delta n_{s}=\operatorname{div}\left(\frac{m_{s} n_{s}}{e_{s} B^{2}} \nabla_{\perp} \phi\right)+\int F_{s} \delta f_{s} d v^{3}
$$

Taking into account equations (23) and (2), the perturbed quasi-neutrality equation reads:

$$
n_{e q} \frac{e}{T_{e}}\left(\phi-\langle\phi\rangle_{F S}\right)=\operatorname{div}\left(\frac{m_{t} n_{t}+m_{k} n_{k}}{e B^{2}} \nabla_{\perp} \phi\right)+\int\left[Z_{t} F_{t} \delta f_{t}+Z_{k} F_{k} \delta f_{k}\right] d v^{3}
$$

Normalizing the electrostatic potential to $T_{t} / e$, defining $\rho_{s}^{2}=\frac{m_{s} T_{t}}{e^{2} B^{2}}$ where the subscript $s$ stands for either $k$ or $t$, neglecting the equilibrium spatial variations with regard to the perturbation spatial variations, and dividing by $n_{e q}$, the quasi-neutrality equation can be recast as:

$$
\frac{T_{t}}{T_{e}}\left(\phi-\langle\phi\rangle_{F S}\right)=\left[\frac{n_{t}}{n_{e q}} \rho_{t}^{2}+\frac{n_{k}}{n_{e q}} \rho_{k}^{2}\right] \nabla_{\perp}^{2} \phi+\frac{1}{n_{e q}} \int\left[Z_{t} F_{t} \delta f_{t}+Z_{k} F_{k} \delta f_{k}\right] d v^{3}
$$

Using Fourier decompositions (16) and (17), defining $\rho_{i}^{2}=\frac{n_{t}}{n_{e q}} \rho_{t}^{2}+\frac{n_{k}}{n_{e q}} \rho_{k}^{2}$, and assuming (like was done in the gyrokinetic equation calculus) that $\partial_{r}$ is dominant over $\frac{\partial_{\theta}}{r}$, the first two harmonics of the quasi-neutrality equation can be written, at first order in $\rho_{*}$, as: 


$$
\begin{aligned}
\rho_{i}^{2} k_{r}^{2} \phi_{0} & =Z_{t} \int \frac{F_{t}}{n_{e q}} \delta f_{t 0} d v^{3}+Z_{k} \int \frac{F_{k}}{n_{e q}} \delta f_{k 0} d v^{3} \\
\frac{T_{t}}{T_{e}} \phi_{1}^{c} & =Z_{t} \int \frac{F_{t}}{n_{e q}} \delta f_{t 1}^{c} d v^{3}+Z_{k} \int \frac{F_{k}}{n_{e q}} \delta f_{k 1}^{c} d v^{3} \\
\frac{T_{t}}{T_{e}} \phi_{1}^{s} & =Z_{t} \int \frac{F_{t}}{n_{e q}} \delta f_{t 1}^{s} d v^{3}+Z_{k} \int \frac{F_{k}}{n_{e q}} \delta f_{k 1}^{s} d v^{3}
\end{aligned}
$$

Reporting equations (21) and (22) into equations (26) and (28), considering that $-T_{t} \partial_{\varepsilon_{t}} \log F_{t}=1$, and removing the odd terms in $v_{\|}$(which disappear when integrated), we get the following system:

$$
\begin{aligned}
\rho_{i}^{2} k_{r}^{2} \phi_{0}=\frac{Z_{t}^{2}}{2} \phi_{1}^{s} \int \frac{F_{t}}{n_{e q}} \frac{\omega \omega_{g, t}}{\omega^{2}-\omega_{\|}^{2}} d v^{3}+\frac{Z_{t}^{2}}{2} \phi_{0} \int \frac{F_{t}}{n_{e q}} \frac{\omega_{g, t}^{2}}{\omega^{2}-\omega_{\|}^{2}} d v^{3} \\
-\frac{Z_{k}^{2}}{2} \phi_{1}^{s} \int \frac{T_{t} \partial_{\varepsilon_{k}} F_{k}}{n_{e q}} \frac{\omega \omega_{g, k}}{\omega^{2}-\omega_{\|}^{2}} d v^{3}-\frac{Z_{k}^{2}}{2} \phi_{0} \int \frac{T_{t} \partial_{\varepsilon_{k}} F_{k}}{n_{e q}} \frac{\omega_{g, k}^{2}}{\omega^{2}-\omega_{\|}^{2}} d v^{3} \\
\frac{T_{t}}{T_{e}} \phi_{1}^{s}=Z_{t}^{2} \phi_{0} \int \frac{F_{t}}{n_{e q}} \frac{\omega \omega_{g, t}}{\omega^{2}-\omega_{\|}^{2}} d v^{3}+Z_{t}^{2} \phi_{1}^{s} \int \frac{F_{t}}{n_{e q}} \frac{\omega_{\|}^{2}}{\omega^{2}-\omega_{\|}^{2}} d v^{3} \\
-Z_{k}^{2} \phi_{0} \int \frac{T_{t} \partial_{\varepsilon_{k}} F_{k}}{n_{e q}} \frac{\omega \omega_{g, k}}{\omega^{2}-\omega_{\|}^{2}} d v^{3}-Z_{k}^{2} \phi_{1}^{s} \int \frac{T_{t} \partial_{\varepsilon_{k}} F_{k}}{n_{e q}} \frac{\omega_{\|}^{2}}{\omega^{2}-\omega_{\|}^{2}} d v^{3}
\end{aligned}
$$

To solve this system, six integrals need to be computed:

$$
\begin{aligned}
I_{1} & =\int \frac{F_{t}}{n_{e q}} \frac{\omega \omega_{g, t}}{\omega^{2}-\omega_{\|}^{2}} d v^{3} \\
I_{2} & =\int \frac{F_{t}}{n_{e q}} \frac{\omega_{g, t}^{2}}{\omega^{2}-\omega_{\|}^{2}} d v^{3} \\
I_{3} & =\int \frac{F_{t}}{n_{e q}} \frac{\omega_{\|}^{2}}{\omega^{2}-\omega_{\|}^{2}} d v^{3} \\
I_{4} & =\int \frac{T_{t} \partial_{\varepsilon_{k}} F_{k}}{n_{e q}} \frac{\omega \omega_{g, k}}{\omega^{2}-\omega_{\|}^{2}} d v^{3} \\
I_{5} & =\int \frac{T_{t} \partial_{\varepsilon_{k}} F_{k}}{n_{e q}} \frac{\omega_{g, k}^{2}}{\omega^{2}-\omega_{\|}^{2}} d v^{3} \\
I_{6} & =\int \frac{T_{t} \partial_{\varepsilon_{k}} F_{k}}{n_{e q}} \frac{\omega_{\|}^{2}}{\omega^{2}-\omega_{\|}^{2}} d v^{3}
\end{aligned}
$$

where $I_{1}, I_{2}$ and $I_{3}$ are integrals over the thermal distribution function, while $I_{4}, I_{5}$ and $I_{6}$ are integrals over the kinetic distribution function.

Regarding the three integrals over the thermal distribution function, we find after calculus:

$$
\begin{aligned}
& I_{1}=-\frac{n_{t}}{n_{e q}} \frac{\sqrt{T_{t} m_{t}}}{e_{t} B} \frac{q k_{r}}{\sqrt{2}}\left[\mathcal{Z}\left(\Omega_{t}\right)\left(1+2 \Omega_{t}^{2}\right)+2 \Omega_{t}\right] \\
& I_{2}=-\frac{n_{t}}{n_{e q}} \frac{T_{t} m_{t}}{e_{t}^{2} B^{2}} q^{2} k_{r}^{2}\left[\mathcal{Z}\left(\Omega_{t}\right)\left(\frac{1}{\Omega_{t}}+2 \Omega_{t}+2 \Omega_{t}^{3}\right)+3+2 \Omega_{t}^{2}\right] \\
& I_{3}=-\frac{n_{t}}{n_{e q}}\left[1+\Omega_{t} \mathcal{Z}\left(\Omega_{t}\right)\right]
\end{aligned}
$$

where $\Omega_{t}$ is the frequency of the mode normalized as follows:

$$
\Omega_{t}=\sqrt{\frac{m_{t}}{2 T_{t}}} q R \omega
$$

Regarding the three integrals over the kinetic distribution function, the derivative of the kinetic distribution function according the the kinetic energy reads:

$$
\begin{aligned}
\partial_{\varepsilon_{k}} F_{k} & =-\frac{n_{k}}{2 T_{t} \tau_{k}}\left(\frac{m_{k}}{2 \pi T_{t} \tau_{k}}\right)^{\frac{3}{2}}\left[e^{-\frac{m_{k}\left(v_{\|}-\bar{v}_{\|}\right)^{2}+2 \mu_{k} B}{2 T_{t} \tau_{k}}}+e^{-\frac{m_{k}\left(v_{\|}+\bar{v}_{\|}\right)^{2}+2 \mu_{k} B}{2 T_{t} \tau_{k}}}\right] \\
& +\frac{n_{k}}{2 T_{t} \tau_{k}} \frac{\bar{v}_{\|}}{v_{\|}}\left(\frac{m_{k}}{2 \pi T_{t} \tau_{k}}\right)^{\frac{3}{2}}\left[e^{-\frac{m_{k}\left(v_{\|}-\bar{v}_{\|}\right)^{2}+2 \mu_{k} B}{2 T_{t} \tau_{k}}}-e^{-\frac{m_{k}\left(v_{\|}+\bar{v}_{\|}\right)^{2}+2 \mu_{k} B}{2 T_{t} \tau_{k}}}\right]
\end{aligned}
$$


We find after calculus:

$$
\begin{aligned}
I_{4}= & \frac{n_{k}}{2 n_{e q}} \frac{\sqrt{T_{t} m_{k}}}{e_{k} B} \frac{\sqrt{2} q k_{r}}{\sqrt{\tau_{k}}}\left\{\left[\mathcal{Z}\left(\frac{\Omega_{k}-\bar{u}_{\|}}{\sqrt{\tau_{k}}}\right)\left[1-\frac{\bar{u}_{\|}}{\Omega_{k}}\right]+\mathcal{Z}\left(\frac{\Omega_{k}+\bar{u}_{\|}}{\sqrt{\tau_{k}}}\right)\left[1+\frac{\bar{u}_{\|}}{\Omega_{k}}\right]\right]\left[\frac{\Omega_{k}^{2}}{\tau_{k}}+\frac{1}{2}\right]\right. \\
& \left.+2 \frac{\Omega_{k}}{\sqrt{\tau_{k}}}-\frac{\bar{u}_{\|}}{\Omega_{k}} \mathcal{Z}\left(\frac{\bar{u}_{\|}}{\sqrt{\tau_{k}}}\right)\right\} \\
I_{5}= & \frac{n_{k}}{2 n_{e q}} \frac{T_{t} m_{k}}{e_{k}^{2} B^{2}} 2 q^{2} k_{r}^{2}\left\{\left[\mathcal{Z}\left(\frac{\Omega_{k}-\bar{u}_{\|}}{\sqrt{\tau_{k}}}\right)\left[1-\frac{\bar{u}_{\|}}{\Omega_{k}}\right]+\mathcal{Z}\left(\frac{\Omega_{k}+\bar{u}_{\|}}{\sqrt{\tau_{k}}}\right)\left[1+\frac{\bar{u}_{\|}}{\Omega_{k}}\right]\right]\left[\frac{\Omega_{k}^{3}}{\tau_{k}^{3 / 2}}+\frac{\Omega_{k}}{\sqrt{\tau_{k}}}+\frac{\sqrt{\tau_{k}}}{2 \Omega_{k}}\right]\right. \\
& \left.+2 \frac{\Omega_{k}^{2}}{\tau_{k}}+3-\frac{\bar{u}_{\|} \sqrt{\tau_{k}}}{\Omega_{k}^{2}} \mathcal{Z}\left(\frac{\bar{u}_{\|}}{\sqrt{\tau_{k}}}\right)\right\} \\
I_{6}= & \frac{n_{k}}{2 n_{e q}} \frac{1}{\tau_{k}}\left\{\mathcal{Z}\left(\frac{\Omega_{k}-\bar{u}_{\|}}{\sqrt{\tau_{k}}}\right) \frac{\Omega_{k}}{\sqrt{\tau_{k}}}\left[1-\frac{\bar{u}_{\|}}{\Omega_{k}}\right]+\mathcal{Z}\left(\frac{\Omega_{k}+\bar{u}_{\|}}{\sqrt{\tau_{k}}}\right) \frac{\Omega_{k}}{\sqrt{\tau_{k}}}\left[1+\frac{\bar{u}_{\|}}{\Omega_{k}}\right]+2\right\}
\end{aligned}
$$

where $\Omega_{k}$ is the frequency of the mode, normalized as follows:

$$
\Omega_{k}=\sqrt{\frac{m_{k}}{2 T_{t}}} q R \omega
$$

and $\bar{u}_{\|}$is the position of the bump-on-tail in the $v_{\|}$direction, normalized as follows:

$$
\bar{u}_{\|}=\sqrt{\frac{m_{k}}{2 T_{t}}} \bar{v}_{\|}
$$

Reporting those expressions of $I_{1}$ to $I_{6}$ into equations (29) and (30), and using a linear combination of those two equations to get rid of $\phi_{1}^{s}$, one gets the dispersion relation with $\phi_{0}$ in factor. When the mode exists, $\phi_{0}$ is non null, which means that it can be removed from the relation. We thus get the following dispersion relation:

$$
\frac{1}{q^{2}}+A_{1}\left(\Omega_{t}\right)+A_{2}\left(\Omega_{k}\right)-\frac{\left[N_{1}\left(\Omega_{t}\right)+N_{2}\left(\Omega_{k}\right)\right]^{2}}{D_{1}\left(\Omega_{t}\right)+D_{2}\left(\Omega_{k}\right)}=0
$$

with:

$$
\begin{aligned}
& A_{1}\left(\Omega_{t}\right)=\frac{n_{e q}}{n_{t}+n_{k} \frac{m_{k}}{m_{t}}} \frac{n_{t}}{n_{e q}}\left\{\mathcal{Z}\left(\Omega_{t}\right)\left[\Omega_{t}^{3}+\Omega_{t}+\frac{1}{2 \Omega_{t}}\right]+\Omega_{t}^{2}+\frac{3}{2}\right\} \\
& A_{2}\left(\Omega_{k}\right)=\frac{m_{k}}{m_{t}} \frac{n_{e q}}{n_{t}+n_{k} \frac{m_{k}}{m_{t}}} \frac{n_{k}}{2 n_{e q}}\left\{\left[\mathcal{Z}\left(\frac{\Omega_{k}-\bar{u}_{\|}}{\sqrt{\tau_{k}}}\right)\left[1-\frac{\bar{u}_{\|}}{\Omega_{k}}\right]+\mathcal{Z}\left(\frac{\Omega_{k}+\bar{u}_{\|}}{\sqrt{\tau_{k}}}\right)\left[1+\frac{\bar{u}_{\|}}{\Omega_{k}}\right]\right]\left[\frac{\Omega_{k}^{3}}{\tau_{k}^{3 / 2}}+\frac{\Omega_{k}}{\sqrt{\tau_{k}}}+\frac{\sqrt{\tau_{k}}}{2 \Omega_{k}}\right]\right. \\
& \left.+2 \frac{\Omega_{k}^{2}}{\tau_{k}}+3-\frac{\bar{u}_{\| \sqrt{\tau_{k}}}}{\Omega_{k}^{2}} \mathcal{Z}\left(\frac{\bar{u}_{\|}}{\sqrt{\tau_{k}}}\right)\right\} \\
& N_{1}\left(\Omega_{t}\right)=Z_{t} \sqrt{\frac{n_{e q}}{n_{t}+n_{k} \frac{m_{k}}{m_{t}}}} \frac{n_{t}}{n_{e q}}\left\{\mathcal{Z}\left(\Omega_{t}\right)\left[\Omega_{t}^{2}+\frac{1}{2}\right]+\Omega_{t}\right\} \\
& N_{2}\left(\Omega_{k}\right)=Z_{k} \frac{1}{\sqrt{\tau_{k}}} \sqrt{\frac{m_{k}}{m_{t}}} \sqrt{\frac{n_{e q}}{n_{t}+n_{k} \frac{m_{k}}{m_{t}}}} \frac{n_{k}}{2 n_{e q}}\left\{\left[\mathcal{Z}\left(\frac{\Omega_{k}-\bar{u}_{\|}}{\sqrt{\tau_{k}}}\right)\left[1-\frac{\bar{u}_{\|}}{\Omega_{k}}\right]+\mathcal{Z}\left(\frac{\Omega_{k}+\bar{u}_{\|}}{\sqrt{\tau_{k}}}\right)\left[1+\frac{\bar{u}_{\|}}{\Omega_{k}}\right]\right]\left[\frac{\Omega_{k}^{2}}{\tau_{k}}+\frac{1}{2}\right]\right. \\
& \left.+2 \frac{\Omega_{k}}{\sqrt{\tau_{k}}}-\frac{\bar{u}_{\|}}{\Omega_{k}} \mathcal{Z}\left(\frac{\bar{u}_{\|}}{\sqrt{\tau_{k}}}\right)\right\} \\
& D_{1}\left(\Omega_{t}\right)=Z_{t}^{2} \frac{n_{t}}{n_{e q}}\left\{\mathcal{Z}\left(\Omega_{t}\right) \Omega_{t}+1\right\}+\frac{T_{t}}{T_{e}} \\
& D_{2}\left(\Omega_{k}\right)=\frac{Z_{k}^{2}}{\tau_{k}} \frac{n_{k}}{2 n_{e q}}\left\{\mathcal{Z}\left(\frac{\Omega_{k}-\bar{u}_{\|}}{\sqrt{\tau_{k}}}\right) \frac{\Omega_{k}}{\sqrt{\tau_{k}}}\left[1-\frac{\bar{u}_{\|}}{\Omega_{k}}\right]+\mathcal{Z}\left(\frac{\Omega_{k}+\bar{u}_{\|}}{\sqrt{\tau_{k}}}\right) \frac{\Omega_{k}}{\sqrt{\tau_{k}}}\left[1+\frac{\bar{u}_{\|}}{\Omega_{k}}\right]+2\right\}
\end{aligned}
$$




\section{REFERENCES}

${ }^{1}$ N. Winsor, J. L. Johnson, and J. M. Dawson, Physics of Fluids (1958-1988) 11, 2448 (1968).

${ }^{2}$ K. Hallatschek and D. Biskamp, Physical Review Letters 86, 1223 (2001).

${ }^{3}$ G. D. Conway, C. Angioni, F. Ryter, P. Sauter, and J. Vicente (ASDEX Upgrade Team), Phys. Rev. Lett. 106, 065001 (2011).

${ }^{4}$ M. Xu, G. R. Tynan, P. H. Diamond, P. Manz, C. Holland, N. Fedorczak, S. C. Thakur, J. H. Yu, K. J. Zhao, J. Q. Dong,

J. Cheng, W. Y. Hong, L. W. Yan, Q. W. Yang, X. M. Song, Y. Huang, L. Z. Cai, W. L. Zhong, Z. B. Shi, X. T. Ding, X. R. Duan, and Y. Liu (HL-2A team), Phys. Rev. Lett. 108, 245001 (2012).

${ }^{5}$ H. Berk, C. Boswell, D. Borba, A. Figueiredo, T. Johnson, M. Nave, S. Pinches, S. Sharapov, and J. E. contributors, Nuclear Fusion 46, S888 (2006).

${ }^{6}$ C. Boswell, H. Berk, D. Borba, T. Johnson, S. Pinches, and S. Sharapov, Physics Letters A 358, 154 (2006).

${ }^{7}$ R. Nazikian, G. Y. Fu, M. E. Austin, H. L. Berk, R. V. Budny, N. N. Gorelenkov, W. W. Heidbrink, C. T. Holcomb, G. J. Kramer, G. R. McKee, M. A. Makowski, W. M. Solomon, M. Shafer, E. J. Strait, and M. A. V. Zeeland, Phys. Rev. Lett. 101, 185001 (2008).

${ }^{8}$ H. Berk and T. Zhou, Nuclear Fusion 50, 035007 (2010).

${ }^{9}$ G. Y. Fu, Physical Review Letters 101, 185002 (2008).

${ }^{10}$ Z. Qiu, F. Zonca, and L. Chen, Plasma Physics and Controlled Fusion 52, 095003 (2010).

${ }^{11}$ Y. I. Kolesnichenko, B. S. Lepiavko, and V. V. Lutsenko, Plasma Physics and Controlled Fusion 55, 125007 (2013).

${ }^{12}$ D. Zarzoso, X. Garbet, Y. Sarazin, R. Dumont, and V. Grandgirard, Physics of Plasmas (1994-present) 19, 022102 (2012).

${ }^{13}$ Y. Sarazin, V. Grandgirard, G. Dif-Pradalier, E. Fleurence, X. Garbet, P. Ghendrih, P. Bertrand, N. Besse, N. Crouseilles, E. Sonnendrücker, G. Latu, and E. Violard, Plasma Physics and Controlled Fusion 48, B179 (2006).

${ }^{14} \mathrm{~V}$. Grandgirard, Y. Sarazin, X. Garbet, G. Dif-Pradalier,
P. Ghendrih, N. Crouseilles, G. Latu, E. Sonnendrücker, N. Besse, and P. Bertrand, Communications in Nonlinear Science and Numerical Simulation 13, 81 (2008), vlasovia 2006: The Second International Workshop on the Theory and Applications of the Vlasov Equation.

${ }^{15}$ Y. Sarazin, V. Grandgirard, J. Abiteboul, S. Allfrey, X. Garbet, P. Ghendrih, G. Latu, A. Strugarek, and G. Dif-Pradalier, Nuclear Fusion 50, 054004 (2010).

${ }^{16}$ D. Zarzoso, Y. Sarazin, X. Garbet, R. Dumont, A. Strugarek, J. Abiteboul, T. Cartier-Michaud, G. Dif-Pradalier, P. Ghendrih, V. Grandgirard, G. Latu, C. Passeron, and O. Thomine, Phys. Rev. Lett. 110, 125002 (2013).

${ }^{17}$ R. Fisher, D. Pace, G. Kramer, M. V. Zeeland, R. Nazikian, W. Heidbrink, and M. García-Muñoz, Nuclear Fusion 52, 123015 (2012).

${ }^{18}$ B. D. Fried and S. D. Conte, The Plasma Dispersion Function (Academic Press, 1961).

${ }^{19}$ F. Zonca and L. Chen, EPL (Europhysics Letters) 83, 35001 (2008).

20 A. Elfimov, A. Smolyakov, and R. Galvão, Physics Letters A 378, 800 (2014).

${ }^{21}$ D. Zarzoso, A. Biancalani, A. Bottino, P. Lauber, E. Poli, J.B. Girardo, X. Garbet, and R. Dumont, Sumbitted to Nuclear Fusion (2014).

${ }^{22}$ S. Jolliet, A. Bottino, P. Angelino, R. Hatzky, T. Tran, B. Mcmillan, O. Sauter, K. Appert, Y. Idomura, and L. Villard, Computer Physics Communications 177, 409 (2007).

${ }^{23}$ A. Bottino, T. Vernay, B. Scott, S. Brunner, R. Hatzky, S. Jolliet, B. F. McMillan, T. M. Tran, and L. Villard, Plasma Physics and Controlled Fusion 53, 124027 (2011)

${ }^{24}$ A. Biancalani, A. Bottino, P. Lauber, and D. Zarzoso, To appear in Nuclear Fusion 54 (2014).

${ }^{25}$ H. Sugama and T.-H. Watanabe, Journal of Plasma Physics 72 , 825 (2006).

${ }^{26}$ H. Sugama and T.-H. Watanabe, Journal of Plasma Physics 74, 139 (2008).

${ }^{27}$ H. S. Zhang and Z. Lin, Physics of Plasmas (1994-present) 17, 072502 (2010). 